\title{
MINIMIZING MOVEMENT FOR A FRACTIONAL POROUS MEDIUM EQUATION IN A PERIODIC SETTING
}

\author{
Lucas C. F. Ferreira, Matheus C. Santos and Julio C. Valencia-Guevara
}

\begin{abstract}
We consider a fractional porous medium equation that extends the classical porous medium and fractional heat equations. The flow is studied in the space of periodic probability measures endowed with a non-local transportation distance constructed in the spirit of the Benamou-Brenier formula. For initial periodic probability measures, we show the existence of absolutely continuous curves that are generalized minimizing movements associated to Rényi entropy. For that, we need to obtain entropy and distance properties and to develop a subdifferential calculus in our setting.
\end{abstract}

\section{INTRODUCTION}

We are concerned with the fractional porous medium equation (FPME)

$$
\partial_{t} \rho+(-\Delta)^{\sigma} \rho^{m}=0 \text { and } \rho(0, x)=\rho_{0}(x)
$$

where the spatial-dimension $d \geq 1,0<\sigma<1$ and $m_{*}:=\frac{(d-2 \sigma)_{+}}{d}<m \leqslant 2$. Our aim is to study the global flow $\rho=\rho_{t}(x)$ with spatial-periodic conditions, i.e. $(t, x) \in[0,+\infty) \times \mathbb{T}^{d}$ ( $\mathbb{T}^{d}$ is the $d$-dimensional torus). We use an optimal transport approach on the space $\mathcal{P}\left(\mathbb{T}^{d}\right)$ equipped with a pseudo-metric $\mathcal{W}$ to find a generalized minimizing movement curve associated to the Rényi entropy, which gives us a weak notion of gradient flow for (1.1).

The equation (1.1) appears in the study of hydrodynamic limits of particle systems [18, the boundary heat control problems as studied by Athanasopoulos and Caffarelli [5], and the so-called anomalous diffusion process that appears when jump processes are introduced in the modeling (see 1, 23, 24). In 11, 12, the authors studied the model (1.1) and developed a theory for the problem in $\mathbb{R}^{d}$ and with general initial data $\rho_{0} \in L^{1}\left(\mathbb{R}^{d}\right)$ that includes also sign changing solutions. For the case $\sigma=1 / 2$ they constructed a weak solution by rewriting the non-local problem in a local way by mean of a well known harmonic extension. The general case was treated in 12 by approximating the initial data by bounded functions and approximating $\mathbb{R}^{d}$ by bounded domains. Existence and uniqueness results are true for any positive value $m$ of the nonlinearity if the initial data is, for example, also bounded, but the full theory only works for values of $m$ larger than the critical value $m_{*}$. In this case, if we take a nonnegative integrable initial data $\rho_{0}$, then conservation of mass, positivity and regularity hold. Let us observe that, for the sake of coherence, we are assuming $m_{*}<m \leqslant 2$ in order to assure mass and sign conservation for (1.1), however most of arguments and results in this paper hold true for the full range $m \in(0,2]$.

The optimal transport approach was used by 20 in the analysis of solutions to the classical porous medium equation

$$
\partial_{t} \rho=\Delta \rho^{m}=\nabla \cdot\left(\rho \nabla U^{\prime}(\rho)\right), \quad U(\rho)=\frac{1}{m-1} \rho^{m} .
$$

\footnotetext{
Departamento de Matemática - IMECC, Universidade Estadual de Campinas, 13083-859, Campinas-SP, Brazil.

Email addresses: lcff@ime.unicamp.br (LCFF), msantos.ufrgs@gmail.com (MCS), ra099814@ime.unicamp.br (JCV-G)

September 10, 2018
} 
It was showed that solutions to this equation can be interpreted as a gradient flow $t \mapsto \rho_{t}(x) d x$ of probabilities on $\mathcal{P}\left(\mathbb{R}^{d}\right)$ associated to the Rényi entropy

$$
\mathcal{U}_{m}(\rho):=\frac{1}{m-1} \int_{\mathbb{R}^{d}} \rho(x)^{m} d x
$$

with respect to (w.r.t. for short) the $L^{2}$-Wasserstein distance $W_{2}$, which is defined by

$$
W_{2}\left(\mu_{0}, \mu_{1}\right):=\inf _{\pi \in \Gamma\left(\mu_{0}, \mu_{1}\right)}\left(\int_{\mathbb{R}^{d} \times \mathbb{R}^{d}}|x-y|^{2} d \pi(x, y)\right)^{1 / 2}, \text { for all } \mu_{0}, \mu_{1} \in \mathcal{P}\left(\mathbb{R}^{d}\right),
$$

where $\Gamma\left(\mu_{0}, \mu_{1}\right)$ is the set of probability measures $\pi$ on $\mathbb{R}^{d} \times \mathbb{R}^{d}$ with marginals $\mu_{0}$ and $\mu_{1}$. This method has allowed one to obtain uniqueness, well-posedness and asymptotic behavior of solutions (such as contraction property and rates for the entropy decay) to the porous medium and to a number of other equations (see e.g. [2, 22, 4, 19]). See also [10, 17] and 16] for periodic solutions of PDEs without fractional Laplacian. Besides that, the use of the entropy and the optimal transport distance also have provided a powerful method to prove existence of solutions by the so-called Minimizing Movement Scheme (in the general metric framework as considered in [4]) or JKO-scheme (in the Wasserstein space $\mathcal{P}_{2}\left(\mathbb{R}^{d}\right)$ used in [19, 4, 9, 13, 7] and many others). In the metric approach, that is obtained in the following way (see [4): if $(X, d)$ is a complete metric space and we consider a lower semicontinuous functional $\mathcal{E}: X \rightarrow(-\infty, \infty]$, then for an initial data $u_{0} \in X$ and time step $\tau>0$, we can consider the implicit discrete scheme defined by

$$
\left\{\begin{array}{l}
u^{0}:=u_{0} \\
u^{n} \in \arg \min \left(\mathcal{E}(u)+\frac{d^{2}\left(u, u^{n-1}\right)}{2 \tau}\right), n \geqslant 1
\end{array}\right.
$$

that is, $u^{n}$ is any minimizer for the functional $u \mapsto \mathcal{E}(u)+\frac{d^{2}\left(u, u^{n-1}\right)}{2 \tau}$. Defining the piecewise constant curve $u_{\tau}(t)=u^{n}$ if $t \in[n \tau,(n+1) \tau)$, then a limit curve $u:[0, \infty) \rightarrow X$ of $u_{\tau}$ as $\tau \rightarrow 0$ (up to a subsequence) is called a generalized minimizing movement curve (GMM curve) starting from $u_{0}$ and it is a weak notion of gradient flow for $\mathcal{E}$.

A slightly different notion of gradient flow in metric spaces involves the concept of curves of maximal slope w.r.t. upper gradient (see [4]): we say that a function $g$ defined on the metric space is an upper gradient for the entropy $\mathcal{E}$ if, for all absolutely continuous curve $v:[0, \infty) \mapsto X$, one has $\left|(\mathcal{E} \circ v)^{\prime}(t)\right| \leqslant$ $g(v(t))\left|v^{\prime}\right|(t)$, where $\left|v^{\prime}\right|(t)$ denotes the metric derivative. And we say that an absolutely continuous curve $u:[0, \infty) \mapsto X$ is a curve of maximal slope for $\mathcal{E}$ w.r.t the upper gradient $g$ if

$$
\frac{d}{d t} \mathcal{E}(u(t)) \leq-\frac{g(u(t))^{2}}{2}-\frac{\left|u^{\prime}\right|^{2}(t)}{2}, \text { for almost } t \in[0, \infty)
$$

Let us comment that if we consider the Wasserstein space $\left(\mathcal{P}_{2}\left(\mathbb{R}^{d}\right), W_{2}\right)$, then the inequality (1.4) becomes essentially equivalent to the continuity equation of the curve $u(t)$. In the most of cases this continuity equation becomes the partial differential equation that gives birth to the functional considered. Another way to explain the relation between the equation (1.2) and the Wasserstein distance (specially in the case where the velocity field is a gradient vector field) can be seen by the dynamic characterization of $W_{2}$, which is known as Benamou-Brenier formula (see [6]):

$$
W_{2}\left(\bar{\mu}_{0}, \bar{\mu}_{1}\right)^{2}=\inf _{(\mu, v) \in \mathcal{C} \mathcal{E}_{1}\left(\bar{\mu}_{0}, \bar{\mu}_{1}\right)}\left\{\int_{0}^{1} \int_{\mathbb{R}^{d}}\left|\nabla v_{t}(x)\right|^{2} d \mu_{t}(x) d t\right\}
$$

where $\mathcal{C} \mathcal{E}_{1}\left(\bar{\mu}_{0}, \bar{\mu}_{1}\right)$ is the set of all sufficiently smooth pairs $\left(\mu_{t}, v_{t}\right)_{t \in[0,1]}$ such that

$$
\left\{\begin{array}{l}
\partial_{t} \mu_{t}+\nabla \cdot\left(\mu_{t} \nabla v_{t}\right)=0, \quad \text { in }(0,1) \times \mathbb{R}^{d} \\
\mu_{0}=\bar{\mu}_{0}, \quad \mu_{1}=\bar{\mu}_{1}
\end{array}\right.
$$

As in [14, 15, we use these ideas to define a metric, in a Benamou-Brenier style, on a subset of $\mathcal{P}\left(\mathbb{T}^{d}\right)$ that incorporates the non-local character of the problem (1.1) and we use it to construct a gradient flow 
associated to the Rényi entropy. For that matter, we need to obtain properties for the non-local metric and entropy, and develop a subdifferential calculus in our setting.

In [15, the author showed that solutions to the fractional linear heat equation (i.e. $m=1$ )

$$
\partial_{t} \rho+(-\Delta)^{\sigma} \rho=0 \text { in }(0, \infty) \times \mathbb{R}^{d} \text { and } \rho(0, x)=\rho_{0}
$$

can be seen as a gradient flow in a non-local metric (built from a Lévy jump kernel) by using, among others, that (1.5) generates a well-behaved semigroup. Due to the presence of $(-\Delta)^{\sigma}$ in (1.1), we are led to consider some ideas from [15. However, we adopt a periodic setting that allows us to obtain the necessary compactness and convergence of the discrete scheme (1.3). So, we are moving in the opposite direction from that of [15] in the sense that we are constructing a flow for (1.1) via the scheme (1.3), and not using previously known information about existence of solutions.

Let us just mention that we consider the $d$-dimensional torus $\mathbb{T}^{d}$ as the cube $[0,1)^{d}$ equipped with the metric $d_{\mathbb{T}^{d}}$ defined by

$$
d_{\mathbb{T}^{d}}(x, y)=\min _{k \in \mathbb{Z}^{d}}|x-y+k| .
$$

From [21] we know that, if $f: \mathbb{T}^{d} \rightarrow \mathbb{R}$ is sufficiently smooth, the following pointwise formula holds for the fractional Laplacian on the torus:

$$
(-\Delta)^{\sigma} f(x)=p \cdot v \cdot \int_{[0,1)^{d}}(f(x)-f(y)) K^{\sigma}(x-y) d y
$$

where the kernel $K^{\sigma}$ is $\mathbb{Z}^{d}$-periodic and defined by

$$
K^{\sigma}(x):=C_{d, \sigma} \sum_{k \in \mathbb{Z}^{d}} \frac{1}{|x+k|^{d+2 \sigma}}, \quad \text { with } C_{d, \sigma}:=\frac{4^{\sigma} \Gamma\left(\frac{d}{2}+\sigma\right)}{\pi^{d / 2}|\Gamma(-\sigma)|} .
$$

Motivated by that, let us call $\rho:(0, \infty) \times \mathbb{T}^{d} \rightarrow \mathbb{R}$ (in some suitable class) a weak solution to (1.1) if, for all $\varphi \in C_{c}^{\infty}\left((0, T) ; C^{\infty}\left(\mathbb{T}^{d}\right)\right)$, one has

$$
\int_{0}^{T} \int_{[0,1)^{d}} \partial_{t} \varphi_{t}(x) \rho_{t} d x d t-\frac{1}{2} \int_{0}^{T} \int_{G\left([0,1)^{d}\right)}\left(\varphi_{t}(x)-\varphi_{t}(y)\right)\left(\rho_{t}(x)^{m}-\rho_{t}(y)^{m}\right) K^{\sigma}(x-y) d x d y d t=0
$$

where

$$
G\left([0,1)^{d}\right):=\left\{(x, y) \in[0,1)^{d} \times[0,1)^{d} \mid x \neq y\right\} .
$$

Throughout the paper, we denote by $\bar{\nabla} f$ the discrete gradient

$$
\bar{\nabla} f(x, y):=f(y)-f(x), \text { for all } f: \mathbb{R}^{d} \rightarrow \mathbb{R} .
$$

Thus, if $m \neq 1$, we can rewrite (1.7) as

$$
\begin{aligned}
\int_{0}^{T} \int_{[0,1)^{d}} \partial_{t} \varphi_{t}(x) \rho_{t} d x d t & =\frac{1}{2} \int_{0}^{T} \int_{G\left([0,1)^{d}\right)} \bar{\nabla} \varphi_{t} \bar{\nabla} \rho_{t}^{m} K^{\sigma}(x-y) d x d y d t \\
& =\frac{1}{2} \int_{0}^{T} \int_{G\left([0,1)^{d}\right)} \bar{\nabla} \varphi_{t} \bar{\nabla} \rho_{t}^{m-1} \frac{\bar{\nabla} \rho_{t}^{m}}{\bar{\nabla} \rho_{t}^{m-1}} K^{\sigma}(x-y) d x d y d t \\
& =\frac{1}{2} \int_{0}^{T} \int_{G\left([0,1)^{d}\right)} \bar{\nabla} \varphi_{t} \bar{\nabla} U_{m}^{\prime}\left(\rho_{t}\right) \widehat{\rho}_{m} K^{\sigma}(x-y) d x d y d t
\end{aligned}
$$

where

$$
U_{m}(s):=\frac{s^{m}}{m-1} \text { and } \widehat{\rho}_{m}(x, y):=\frac{m-1}{m} \frac{\bar{\nabla} \rho^{m}(x, y)}{\bar{\nabla} \rho^{m-1}(x, y)} .
$$


If $m=1$ we have the same representation if we define

$$
U_{1}(s):=s \log s \text { and } \widehat{\rho}_{1}(x, y):=\frac{\bar{\nabla} \rho(x, y)}{\bar{\nabla} \log \rho(x, y)} .
$$

Therefore, we can write (1.10) formally as

$$
\partial_{t} \rho-\bar{\nabla} \cdot\left(\widehat{\rho_{m}} \bar{\nabla} U_{m}^{\prime}(\rho)\right)=0
$$

which resembles the classical porous medium equation given in (1.2).

This paper is organized as follows. In Section 2 we give some basic definitions and notations. Section 3 is devoted to the action functional $\mathcal{A}(\mu, \boldsymbol{\nu})$ and its properties. Some results about a periodic non-local continuity equation are presented in Section 4. In Section 5 we define the periodic non-local Wasserstein distance $\mathcal{W}$ based on the Benamou-Brenier formula and show some of its properties. In Section 6 a subdifferential calculus is constructed in the periodic Wasserstein space $\mathcal{P}\left(\mathbb{T}^{d}\right)$ endowed with the nonlocal metric $\mathcal{W}$. In Section 7 we show the existence of GMM curves associated to (1.1).

\section{Notation AND PRELIMINARIES}

In this section, we make some definitions and remarks about the notation that will be used in the sequel.

Following [17, we define the equivalence relation in $\mathcal{P}\left(\mathbb{R}^{d}\right)$ given as

$$
\mu_{1}, \mu_{2} \in \mathcal{P}\left(\mathbb{R}^{d}\right) . \quad \mu_{1} \sim \mu_{2} \equiv \int_{\mathbb{R}^{d}} \zeta(x) d \mu_{1}(x)=\int_{\mathbb{R}^{d}} \zeta(x) d \mu_{2}(x), \forall \zeta \in C\left(\mathbb{T}^{d}\right),
$$

where $C\left(\mathbb{T}^{d}\right)$ stands for the space of continuous functions in $\mathbb{R}^{d}$ which are $\mathbb{Z}^{d}$-periodic. Then, it is well known that $\mathcal{P}\left(\mathbb{T}^{d}\right)=\mathcal{P}\left(\mathbb{R}^{d}\right) / \sim$.

Considering the set

$$
G\left(\mathbb{R}^{d}\right)=\left\{(x, y) \in \mathbb{R}^{d} \times \mathbb{R}^{d} \mid x \neq y\right\}
$$

and the space of functions

$$
C_{c}\left(G\left(\mathbb{T}^{d}\right)\right)=\left\{\varphi \in C\left(G\left(\mathbb{R}^{d}\right)\right) \mid \min \{|x-y| \mid(x, y) \in \operatorname{supp}(\varphi)\}>0, \varphi \text { is }\left(\mathbb{Z}^{d} \times \mathbb{Z}^{d}\right) \text {-periodic }\right\},
$$

we can analogously define the equivalence relation

$$
\boldsymbol{\nu}_{1}, \boldsymbol{\nu}_{2} \in \mathcal{M}_{0}\left(\mathbb{R}^{d}\right) \quad \boldsymbol{\nu}_{1} \sim \boldsymbol{\nu}_{2} \equiv \int_{G\left(\mathbb{R}^{d}\right)} \varphi(x, y) d \boldsymbol{\nu}_{1}(x, y)=\int_{G\left(\mathbb{R}^{d}\right)} \varphi(x, y) d \boldsymbol{\nu}_{2}(x, y), \forall \varphi \in C_{c}\left(G\left(\mathbb{T}^{d}\right)\right),
$$

where

$$
\mathcal{M}_{0}\left(\mathbb{R}^{d}\right):=\left\{\boldsymbol{\nu} \in \mathcal{M}_{\mathrm{loc}}\left(G\left(\mathbb{R}^{d}\right)\right)\left|\int_{G\left(\mathbb{R}^{d}\right)}(1 \wedge|x-y|) d\right| \boldsymbol{\nu} \mid(x, y)<\infty\right\},
$$

and $\mathcal{M}_{\text {loc }}\left(G\left(\mathbb{R}^{d}\right)\right)$ stands for the locally finite Radon measures on $G\left(\mathbb{R}^{d}\right)$. It is straightforward to check that

$$
\mathcal{M}_{0}\left(\mathbb{T}^{d}\right) \simeq \mathcal{M}_{0}\left(\mathbb{R}^{d}\right) / \sim
$$

where

$$
\mathcal{M}_{0}\left(\mathbb{T}^{d}\right):=\left\{\boldsymbol{\nu} \in \mathcal{M}_{\mathrm{loc}}\left(G\left([0,1)^{d}\right)\right)\left|\int_{G\left([0,1)^{d}\right)} d_{\mathbb{T}^{d}}(x, y) d\right| \boldsymbol{\nu} \mid(x, y)<\infty\right\},
$$

and $\mathcal{M}_{\text {loc }}\left(G\left([0,1)^{d}\right)\right)$ denotes the set of locally finite signed Radon measure defined in the Borelians of $G\left([0,1)^{d}\right)$, defined in (1.8), that are generated by $d_{\mathbb{T}^{d}}$. 
Next, $\forall \zeta \in C\left(\mathbb{T}^{d}\right), \forall[\mu] \in \mathcal{P}\left(\mathbb{T}^{d}\right)$ we define $\int_{\mathbb{T}^{d}} \zeta d[\mu]:=\int_{\mathbb{R}^{d}} \zeta d \mu$. In particular, since there exists only one $\bar{\mu} \in \mathcal{P}\left(\mathbb{R}^{d}\right)$ such that supp $\bar{\mu} \subseteq[0,1)^{d}$ e $\bar{\mu} \sim \mu$ (see [10, 17]), we actually have

$$
\int_{\mathbb{T}^{d}} \zeta d[\mu]=\int_{[0,1)^{d}} \zeta d \bar{\mu} .
$$

The measure $\bar{\mu}$ can be obtained by

$$
\bar{\mu}(B):=\sum_{k \in \mathbb{Z}^{d}} \mu(B+k), \quad \text { for all Borelians } B \subseteq[0,1)^{d},
$$

which is the push-forward of $\mu$ by the map $T: \mathbb{R}^{d} \rightarrow[0,1)$ defined by $T\left(x_{1}, \ldots, x_{d}\right):=\left(x_{1}-\left\lfloor x_{1}\right\rfloor, \ldots, x_{d}-\right.$ $\left\lfloor x_{d}\right\rfloor$ ), where $\lfloor$.$\rfloor is the greatest integer function.$

Analogously, for $\varphi \in C_{c}\left(G\left(\mathbb{T}^{d}\right)\right)$ and $[\boldsymbol{\nu}] \in \mathcal{M}_{0}\left(\mathbb{T}^{d}\right)$ we define

$$
\int_{G\left(\mathbb{T}^{d}\right)} \varphi d[\boldsymbol{\nu}]:=\int_{G\left(\mathbb{R}^{d}\right)} \varphi d \boldsymbol{\nu} .
$$

In particular, the integrability condition in (2.1) allows us to use the push-forward by $T$ and obtain that there exists $\overline{\boldsymbol{\nu}} \in \mathcal{M}_{\mathrm{loc}}\left(G\left(\mathbb{R}^{d}\right)\right)$ such that $\operatorname{supp} \overline{\boldsymbol{\nu}} \subseteq G\left([0,1)^{d}\right)$ and $\overline{\boldsymbol{\nu}} \sim \boldsymbol{\nu}$. Therefore

$$
\int_{\mathbb{T}^{d}} \varphi d[\boldsymbol{\nu}]=\int_{G\left([0,1)^{d}\right)} \varphi d \overline{\boldsymbol{\nu}}
$$

Let us recall the narrow convergence in $\mathcal{P}\left(\mathbb{T}^{d}\right)$ which will be used in the results of compactness. In fact, this topology coincides with the weak-* topology induced by the dual of $C\left(\mathbb{T}^{d}\right)$.

Definition 2.1. We say that a sequence $\left(\mu_{n}\right)_{n} \subseteq \mathcal{P}\left(\mathbb{T}^{d}\right)$ weakly (narrowly) converges to $\mu \in \mathcal{P}\left(\mathbb{T}^{d}\right)$ if

$$
\int_{[0,1)^{d}} \varphi(x) d \mu_{n}(x) \rightarrow \int_{[0,1)^{d}} \varphi(x) d \mu(x), \quad \forall \varphi \in C\left(\mathbb{T}^{d}\right) .
$$

Remark 2.2. Note that the cube $[0,1)^{d}$ with the torus metric $d_{\mathbb{T}^{d}}$ (see (1.6)) is a compact metric space and therefore, by Prokhorov's Theorem, $\mathcal{P}\left(\mathbb{T}^{d}\right)$ is a weakly compact space.

\section{The ACTION FUnCTIONAL}

The weak formulation given in (1.10) for the fractional porous medium equation leads us to the following function, which is used there as a mean for probability densities between any two different points. We define the $m$-mean $\theta_{m}:[0, \infty) \times[0, \infty) \rightarrow[0, \infty)$ by

$$
\theta_{m}(s, t):=\left\{\begin{array}{cl}
\frac{s-t}{\log s-\log t}, & \text { if } m=1 \\
\frac{m-1}{m} \frac{s^{m}-t^{m}}{s^{m-1}-t^{m-1}}, & \text { else, }
\end{array} \text { for } s \neq t,\right.
$$

and extended by continuity to $s=t$. The function $\theta_{m}:[0, \infty)^{2} \rightarrow[0, \infty)$ satisfies the following integral representation

$$
\theta_{m}(s, t)= \begin{cases}\int_{0}^{1} s^{\alpha} t^{1-\alpha} d \alpha, & \text { if } m=1 \\ \int_{0}^{1}\left((1-\alpha) s^{m-1}+\alpha t^{m-1}\right)^{\frac{1}{m-1}} d \alpha, & \text { if } m \neq 1\end{cases}
$$

and the following properties for $0<m \leqslant 2$ : 
1. Symmetry: $\theta_{m}(s, t)=\theta_{m}(t, s)$;

2. Homogeneity: $\theta_{m}(\lambda s, \lambda t)=\lambda \theta_{m}(s, t)$, for all $\lambda>0$;

3. Concavity: $\theta_{m}$ is concave for $0<m \leqslant 2$;

4. Monotonicity: If $m_{1}<m_{2}$ then $\theta_{m_{1}}(s, t)<\theta_{m_{2}}(s, t)$ for all $s, t>0$;

5. Monotonicity: If $0 \leqslant s_{1} \leqslant s_{2}$ and $t \geqslant 0$ then $\theta_{m}\left(s_{1}, t\right) \leqslant \theta_{m}\left(s_{2}, t\right)$;

6. Boundary values: $\theta_{m}(0, t)=0$ for all $t \geqslant 0$ if and only if $m \leqslant 1$. For $1<m \leqslant 2$, we have $\theta_{m}(0, t)=\frac{m-1}{m} t$, for all $t \geqslant 0$.

Given a probability density $\rho(x) d x \in \mathcal{P}\left(\mathbb{T}^{d}\right)$, we use the following notation for the $m$-mean of $\rho$ between any two points $(x, y) \in \mathbb{T}^{d} \times \mathbb{T}^{d}$ :

$$
\widehat{\rho}_{m}(x, y):=\theta_{m}(\rho(x), \rho(y)) .
$$

Let us define now the action functional that will be used in the dynamic definition of the non-local fractional Wasserstein distance. Just for a moment, let us write the torus as the cube $[0,1)^{d}$ with its Borelians defined by the metric $d_{\mathbb{T}^{d}}$.

Definition 3.1. Given $(\mu, \boldsymbol{\nu}) \in \mathcal{P}\left([0,1)^{d}\right) \times \mathcal{M}_{\text {loc }}\left(G\left([0,1)^{d}\right)\right)$, we define $\boldsymbol{\mu}^{1}, \boldsymbol{\mu}^{2} \in \mathcal{M}_{\text {loc }}\left(G\left([0,1)^{d}\right)\right)$ by

$$
d \boldsymbol{\mu}^{1}(x, y)=K^{\sigma}(x-y) d y d \mu(x), \quad d \boldsymbol{\mu}^{2}(x, y)=K^{\sigma}(x-y) d x d \mu(y) \in \mathcal{M}_{l o c}\left(G\left([0,1)^{d}\right)\right)
$$

Now, let $\boldsymbol{\lambda} \in \mathcal{M}_{\text {loc }}\left(G\left([0,1)^{d}\right)\right)$ be a nonnegative Borelian measure such that $\boldsymbol{\mu}^{1}, \boldsymbol{\mu}^{2}$ and $\boldsymbol{\nu}$ are absolutely continuous with respect to $\boldsymbol{\lambda}$ with $d \boldsymbol{\mu}^{1}=\rho^{1} d \boldsymbol{\lambda}, d \boldsymbol{\mu}^{2}=\rho^{2} d \boldsymbol{\lambda}$ and $d \boldsymbol{\nu}=w d \boldsymbol{\lambda}$. The measure $\lambda$ can be taken as $\left|\boldsymbol{\mu}^{1}\right|+\left|\boldsymbol{\mu}^{2}\right|+|\boldsymbol{\nu}|$, for examle. Then we define the action $\mathcal{A}$ by

$$
\mathcal{A}(\mu, \boldsymbol{\nu}):=\frac{1}{2} \int_{G\left([0,1)^{d}\right)} \frac{w(x, y)^{2}}{\theta_{m}\left(\rho^{1}(x, y), \rho^{2}(x, y)\right)} d \boldsymbol{\lambda}(x, y)
$$

Remark 3.2. We should mention that the action functional does depend on the order $\sigma$ of the fractional Laplacian and also on the nonlinearity $m$, although we will not mention them in the notation in order to keep it clearer.

This functional is well defined since the integrand is positively 1-homogeneous.

Let us show that for $m=1$ the present case is the natural periodization of the one defined in [15]. Given $\mu \in \mathcal{P}\left(\mathbb{R}^{d}\right)$, we can define

$$
d \boldsymbol{\mu}^{1}(x, y)=\frac{C_{d, \sigma}}{|x-y|^{d+2 \sigma}} d y d \mu(x) \in \mathcal{M}_{\mathrm{loc}}\left(G\left(\mathbb{R}^{d}\right)\right)
$$

and consider the respective equivalent classes $[\mu]$ and $\left[\boldsymbol{\mu}^{1}\right]$. Therefore, if $\varphi \in C_{c}\left(G\left(\mathbb{T}^{d}\right)\right)$, we can write for all $x \in \mathbb{R}^{d}$

$$
\begin{aligned}
\int_{\mathbb{R}^{d} \backslash\{x\}} \frac{\varphi(x, y)}{|x-y|^{d+2 \sigma}} d y & =\sum_{k \in \mathbb{Z}^{d}} \int_{\left([0,1)^{d}+k\right) \backslash\{x\}} \frac{\varphi(x, y)}{|x-y|^{d+2 \sigma}} d y=\sum_{k \in \mathbb{Z}^{d}} \int_{[0,1)^{d} \backslash\{\bar{x}\}} \frac{\varphi(x, y-k)}{|x-y+k|^{d+2 \sigma}} d y \\
& =\int_{[0,1)^{d} \backslash\{\bar{x}\}} \varphi(x, y) \sum_{k \in \mathbb{Z}^{d}} \frac{1}{|x-y+k|^{d+2 \sigma}} d y \\
& =\frac{1}{C_{d, \sigma}} \int_{[0,1)^{d} \backslash\{\bar{x}\}} \varphi(x, y) K^{\sigma}(x-y) d y
\end{aligned}
$$


and then

$$
\begin{aligned}
\int_{G\left(\mathbb{T}^{d}\right)} \varphi(x, y) d\left[\boldsymbol{\mu}^{1}\right](x, y) & =\int_{G\left(\mathbb{R}^{d}\right)} \varphi(x, y) d \boldsymbol{\mu}^{1}(x, y) \\
& =\int_{\mathbb{R}^{d}} \int_{\mathbb{R}^{d} \backslash\{x\}} \frac{C_{d, \sigma}}{|x-y|^{d+2 \sigma}} \varphi(x, y) d y d \mu(x) \\
& =\int_{\mathbb{R}^{d}} \int_{[0,1)^{d} \backslash\{\bar{x}\}} \varphi(x, y) K^{\sigma}(x-y) d y d \mu(x) \\
& =\int_{[0,1)^{d}} \int_{[0,1)^{d} \backslash\{\bar{x}\}} \varphi(x, y) K^{\sigma}(x-y) d y d \bar{\mu}(x) \\
& =\int_{G\left([0,1)^{d}\right)} \varphi(x, y) K^{\sigma}(x-y) d y d \bar{\mu}(x)
\end{aligned}
$$

Therefore, we can write $d\left[\boldsymbol{\mu}^{1}\right](x, y)=K^{\sigma}(x-y) d y d[\mu](x)$. Analogously we define $d\left[\boldsymbol{\mu}^{2}\right](x, y)=K^{\sigma}(x-$ y) $d x d[\mu](y)$.

From now onwards we denote any equivalence class in $\mathcal{P}\left(\mathbb{T}^{d}\right)$ or $\mathcal{M}_{\text {loc }}\left(G\left(\mathbb{T}^{d}\right)\right)$ by its representative element with support in $[0,1)^{d}$, and thus, we skip the bracket notation. Also, in order to simplify the notation, we will denote by $d K^{\sigma}(x, y)$ the locally finite measure $K^{\sigma}(x-y) d x d y \in \mathcal{M}_{\text {loc }}\left(G\left([0,1)^{d}\right)\right)$.

A key ingredient to working with the action functional and later with the Minimizing Movement Scheme is the lower semi-continuity property. This is a direct consequence of the following more general result that can be found in 8 .

Lemma 3.3. Let $\Omega$ be a locally compact Polish space and $f: \Omega \times \mathbb{R}^{n} \rightarrow[0,+\infty]$ be a lower semicontinuous function such that $f(z, \cdot)$ is convex and positively 1-homogeneous for every $z \in \Omega$. Then the functional $F: \mathcal{M}_{l o c}(\Omega)^{n} \rightarrow[0,+\infty]$ defined by

$$
F\left(\sigma_{1}, \ldots, \sigma_{n}\right)=\int_{\Omega} f\left(z, h_{1}(z), \ldots, h_{n}(z)\right) d \lambda(z)
$$

where $\lambda \in \mathcal{M}_{\text {loc }}(\Omega)$ is such that $d \sigma_{i}=h_{i}(z) d \lambda$, for $1 \leqslant i \leqslant n$, is weak ${ }^{*}$ lower semicontinuous on $\mathcal{M}_{\text {loc }}(\Omega)$.

The results contained in the next lemma are periodic analogous to the ones obtained in [15] for the equivalent problem in $\mathbb{R}^{d}$.

Lemma 3.4. The action functional $\mathcal{A}$ is convex in $\mathcal{P}\left(\mathbb{T}^{d}\right) \times \mathcal{M}_{\text {loc }}\left(G\left(\mathbb{T}^{d}\right)\right)$. Also, it is lower semicontinuous, i.e., if $\mu_{n} \rightarrow \mu$ weakly and $\boldsymbol{\nu}_{n} \rightarrow \boldsymbol{\nu}$ weakly*, then

$$
\mathcal{A}(\mu, \boldsymbol{\nu}) \leqslant \liminf _{n \rightarrow \infty} \mathcal{A}\left(\mu_{n}, \boldsymbol{\nu}_{n}\right) .
$$

Furthermore, for any $d \mu=\rho(x) d x$ and $\boldsymbol{\nu} \in \mathcal{M}_{\text {loc }}\left(G\left(\mathbb{T}^{d}\right)\right)$ such that $\mathcal{A}(\mu, \boldsymbol{\nu})<\infty$, there exists a function $w: G\left([0,1)^{d}\right) \rightarrow \mathbb{R}$ such that $\boldsymbol{\nu}=w \widehat{\rho}_{m} d K^{\sigma}$ and

$$
\mathcal{A}(\mu, \boldsymbol{\nu})=\frac{1}{2} \int_{G\left([0,1)^{d}\right)}|w(x, y)|^{2} \widehat{\rho}_{m}(x, y) d K^{\sigma}(x, y) .
$$

Proof. The convexity is clear from the fact that $\theta$ is concave and $(x, y) \mapsto x^{2} / y$ is convex and decreasing in $y$. The lower semicontinuity follows from Lemma 3.3 for $\Omega=G\left([0,1)^{d}\right), f\left(z, h_{1}, h_{2}, h_{3}\right):=$ $\left|h_{3}\right|^{2} \theta_{m}\left(h_{1}, h_{2}\right)^{-1}$ and noting that $\boldsymbol{\mu}^{i, n} \stackrel{*}{\rightarrow} \boldsymbol{\mu}^{i}$ for $i=1,2$ if $\mu^{n} \rightarrow \mu$. Thus

$$
\mathcal{A}(\mu, \boldsymbol{\nu})=F\left(\boldsymbol{\mu}^{1}, \boldsymbol{\mu}^{2}, \boldsymbol{\nu}\right)=\frac{1}{2} \int_{G\left([0,1)^{d}\right)} f\left(\rho^{1}, \rho^{2}, w\right) d \boldsymbol{\lambda}
$$

is lower semicontinuous w.r.t. the weak-weak* convergence. 
The second claim follows from a similar proof as the one in [15, Lemma 2.3] and it is left to the reader.

The following lemma will be useful in order to provide uniform estimates that will be needed in several results in the sequel.

Lemma 3.5. There exists a constant $C>0$ such that

$$
\int_{G\left([0,1)^{d}\right)} d_{\mathbb{T}^{d}}(x, y)|\boldsymbol{\nu}|(x, y) \leqslant C \sqrt{\mathcal{A}(\mu, \boldsymbol{\nu})},
$$

for all $\mu \in \mathcal{P}\left(\mathbb{T}^{d}\right)$ and $\boldsymbol{\nu} \in \mathcal{M}_{\text {loc }}\left(G\left(\mathbb{T}^{d}\right)\right)$. Also, for every Borelian $F \subseteq G\left(\mathbb{T}^{d}\right)$ such that $\delta:=$ $\inf \left\{d_{\mathbb{T}^{d}}(x, y):(x, y) \in F\right\}>0$, there exists a constant $C=C(\delta)$ such that

$$
|\nu|(F) \leqslant C \sqrt{\mathcal{A}(\mu, \nu)}
$$

Proof. We start by estimating

$$
|\boldsymbol{\nu}|(F)=\frac{1}{\delta} \int_{F} \delta d|\boldsymbol{\nu}| \leqslant \frac{1}{\delta} \int_{F} d(x, y) d|\boldsymbol{\nu}|
$$

Now, let $\boldsymbol{\lambda} \in \mathcal{M}_{\text {loc }}\left(G\left(\mathbb{T}^{d}\right)\right)$ such that $\boldsymbol{\mu}^{1}, \boldsymbol{\mu}^{1}, \boldsymbol{\nu} \ll \boldsymbol{\lambda}$ with $d \boldsymbol{\mu}^{i}=\rho^{i} d \boldsymbol{\lambda}$ for $i=1,2$ and $d \boldsymbol{\nu}=w d \boldsymbol{\lambda}$. If $\mathcal{A}(\mu, \nu)<\infty$ then $\boldsymbol{\lambda}\left(\left\{(x, y) \in G\left(\mathbb{T}^{d}\right) \mid \frac{w^{2}}{\theta_{m}\left(\rho^{1}, \rho^{2}\right)}=\infty\right\}\right)=0$ and thus

$$
\begin{aligned}
\int_{G\left([0,1)^{d}\right)} d_{\mathbb{T}^{d}}(x, y) d|\boldsymbol{\nu}| & =\int_{G\left([0,1)^{d}\right)} d_{\mathbb{T}^{d}}(x, y)|w(x, y)| d \boldsymbol{\lambda} \\
& =\int_{G\left([0,1)^{d}\right)} d_{\mathbb{T}^{d}}(x, y) \sqrt{2 \theta_{m}\left(\rho^{1}, \rho^{2}\right)} \sqrt{\frac{w^{2}}{2 \theta_{m}\left(\rho^{1}, \rho^{2}\right)}} d \boldsymbol{\lambda} \\
& \leqslant\left(2 \int_{G\left([0,1)^{d}\right)} d_{\mathbb{T}^{d}}^{2}(x, y) \theta_{m}\left(\rho^{1}, \rho^{2}\right) d \boldsymbol{\lambda}\right)^{\frac{1}{2}}\left(\int_{G\left([0,1)^{d}\right)} \frac{w^{2}}{2 \theta_{m}\left(\rho^{1}, \rho^{2}\right)} d \boldsymbol{\lambda}\right)^{\frac{1}{2}} \\
& =C \sqrt{\mathcal{A}(\mu, \boldsymbol{\nu})},
\end{aligned}
$$

where $C$ is the constant that appears in the following estimate:

$$
\begin{aligned}
\int_{G\left([0,1)^{d}\right)} d_{\mathbb{T}^{d}}^{2}(x, y) \theta_{m}\left(\rho^{1}, \rho^{2}\right) d \boldsymbol{\lambda} & \leqslant \int_{G\left([0,1)^{d}\right)} d_{\mathbb{T}^{d}}^{2}(x, y) \frac{\rho^{1}+\rho^{2}}{2} d \boldsymbol{\lambda} \\
& \leqslant \frac{1}{2} \int_{G\left([0,1)^{d}\right)} d_{\mathbb{T}^{d}}^{2}(x, y) K^{\sigma}(x-y) d y d \mu(x) \\
& +\frac{1}{2} \int_{G\left([0,1)^{d}\right)} d_{\mathbb{T}^{d}}^{2}(x, y) K^{\sigma}(x-y) d x d \mu(y) \\
& =\int_{G\left([0,1)^{d}\right)} d_{\mathbb{T}^{d}}^{2}(x, y) K^{\sigma}(x-y) d y d \mu(x) \\
& \leqslant \sup _{x \in[0,1)^{d}} \int_{[0,1)^{d}} d_{\mathbb{T}^{d}}^{2}(x, y) K^{\sigma}(x-y) d y=: C .
\end{aligned}
$$


We can see that $C$ is finite by using that $d_{\mathbb{T}^{d}}(x, y) \leqslant \min _{k \in \mathbb{Z}^{d}}\{|x-y+k|\} \leqslant 1$ and

$$
\begin{aligned}
\int_{[0,1)^{d}} d_{\mathbb{T}^{d}}^{2}(x, y) K^{\sigma}(x-y) d y & \leqslant C_{d, \sigma} \int_{[0,1)^{d}} \sum_{|k| \leqslant 1} \frac{1}{|x-y+k|^{d+2 \sigma-2}} d y \\
& +C_{d, \sigma} \int_{[0,1)^{d}} \sum_{|k|>1} \frac{1}{|x-y+k|^{d+2 \sigma}} d y \\
& \leqslant C_{d, \sigma} \int_{|x-y| \leqslant 2 \sqrt{d}} \frac{1}{|x-y|^{d+2 \sigma-2}} d y \\
& +C_{d, \sigma} \int_{|x-y|>2 \sqrt{d}} \frac{1}{|x-y|^{d+2 \sigma}} d y \\
& =C_{d, \sigma} \int_{|y| \leqslant 2 \sqrt{d}} \frac{1}{|y|^{d+2 \sigma-2}} d y+C_{d, \sigma} \int_{|y|>2 \sqrt{d}} \frac{1}{|y|^{d+2 \sigma}} d y \\
& <\infty
\end{aligned}
$$

\section{The NON-LOCAL CONTINUITY EQUATION IN $\mathbb{T}^{d}$}

This section is devoted to present some results about the periodic continuity equation

$$
\partial_{t} \mu_{t}+\bar{\nabla} \cdot \boldsymbol{\nu}_{t}=0, \quad \text { in }(0, T) \times[0,1)^{d},
$$

where $\bar{\nabla}$ is the discret gradient defined in (1.9). Here $\left(\mu_{t}\right)_{t \in[0, T]}$ and $\left(\boldsymbol{\nu}_{t}\right)_{t \in[0, T]}$ are Borel families of measures in $\mathcal{P}\left(\mathbb{T}^{d}\right)$ and $\mathcal{M}_{\text {loc }}\left(G\left(\mathbb{T}^{d}\right)\right)$, respectively, and satisfying

$$
\int_{0}^{T} \int_{G\left([0,1)^{d}\right)} d_{\mathbb{T}^{d}}(x, y) d\left|\boldsymbol{\nu}_{t}\right| d t<\infty .
$$

We suppose that (4.1) holds in the periodic distributional sense, i.e., for all $\varphi \in C_{c}^{\infty}\left((0, T) ; C^{\infty}\left(\mathbb{T}^{d}\right)\right)$ we have that the pair $\left(\mu_{t}, \boldsymbol{\nu}_{t}\right)_{t \in[0, T]}$ satisfies

$$
\int_{0}^{T} \int_{[0,1)^{d}} \partial_{t} \varphi_{t} d \mu_{t}(x) d t+\frac{1}{2} \int_{0}^{T} \int_{G\left([0,1)^{d}\right)} \bar{\nabla} \varphi_{t}(x, y) d \boldsymbol{\nu}_{t}(x, y) d t=0 .
$$

Let us define the class of pairs that we will use in the Benamou-Brenier formulation.

Definition 4.1. Given $\widetilde{\mu}_{0}, \widetilde{\mu}_{1} \in \mathcal{P}\left(\mathbb{T}^{d}\right)$, we denote $\mathcal{C} \mathcal{E}_{T}\left(\widetilde{\mu}_{0}, \widetilde{\mu}_{1}\right)$ the set of pairs $\left(\mu_{t}, \boldsymbol{\nu}_{t}\right)_{t \in[0, T]}$ such that

i) $t \in[0, T] \mapsto \mu_{t}$ is weakly continuous (Definition [2.1) with $\mu_{0}=\widetilde{\mu}_{0}$ and $\mu_{1}=\widetilde{\mu}_{1}$;

ii) $\left(\boldsymbol{\nu}_{t}\right)_{t \in[0, T]}$ is a Borel family in $\mathcal{M}_{l o c}\left(G\left(\mathbb{T}^{d}\right)\right)$ with $\int_{0}^{T} \int_{G\left([0,1)^{d}\right)} d_{\mathbb{T}^{d}}(x, y) d\left|\boldsymbol{\nu}_{t}\right| d t<\infty$;

iii) The pair $\left(\mu_{t}, \boldsymbol{\nu}_{t}\right)_{t \in[0, T]}$ satisfies $\partial_{t} \mu_{t}+\bar{\nabla} \cdot \boldsymbol{\nu}_{t}=0$ in the periodic distributional sense.

Remark 4.2. Let us denote by $\mathcal{C E}_{T}$ all the pairs $\left(\mu_{t}, \boldsymbol{\nu}_{t}\right)_{t \in[0, T]}$ satisfying the items ii) and iii) above, with $t \mapsto \mu_{t}$ weakly continuous but with no fixed end points.

Remark 4.3. It is clear that if a pair $\left(\mu_{t}, \boldsymbol{\nu}_{t}\right)_{t \in[0, T]} \in \mathcal{C} \mathcal{E}_{T}\left(\widetilde{\mu}_{0}, \widetilde{\mu}_{1}\right)$ and $\varphi \in C\left(\mathbb{T}^{d}\right)$ is Lipschitz, then it also satisfies

$$
\int \varphi(x) d \widetilde{\mu}_{1}(x)-\int \varphi(x) d \widetilde{\mu}_{0}(x)=\frac{1}{2} \int_{0}^{1} \int_{G\left([0,1)^{d}\right)} \bar{\nabla} \varphi(x, y) d \boldsymbol{\nu}_{t} d t .
$$


The condition $i$ i) ensures that the weak formulation iii) is well defined since, for every $\varphi \in C\left(\mathbb{T}^{d}\right)$ Lipschitz and $k \in \mathbb{Z}^{d}$ we have

$$
|\bar{\nabla} \varphi(x, y)|=|\varphi(x)-\varphi(y)|=|\varphi(x)-\varphi(y+k)| \leqslant[\varphi]_{1}|x-y-k|
$$

where

$$
[\varphi]_{1}:=\sup _{x \neq y} \frac{|\varphi(x)-\varphi(y)|}{|x-y|}
$$

and therefore $|\bar{\nabla} \varphi(x, y)| \leqslant[\varphi]_{1} d_{\mathbb{T}^{d}}(x, y)$.

The lemma below consists in a time-rescaling property for solutions of 4.1).

Lemma 4.4. Let $\boldsymbol{t}:[0, \bar{T}] \rightarrow[0, T]$ be a strictly increasing absolutely continuous map with absolutely continuous inverse $\boldsymbol{s}:=\boldsymbol{t}^{-1}$. Then $\left(\mu_{t}, \boldsymbol{\nu}_{t}\right)_{t \in[0, T]}$ is a distributional solution of $\partial_{t} \mu_{t}+\bar{\nabla} \cdot \boldsymbol{\nu}_{t}=0$ in $(0, T) \times[0,1)^{d}$ in the periodic sense if and only if the pair $\left(\bar{\mu}_{s}, \overline{\boldsymbol{\nu}}_{s}\right)_{s \in[0, \bar{T}]}$, defined by $\bar{\mu}_{s}=\mu_{\boldsymbol{t}(s)}$ and $\overline{\boldsymbol{\nu}}_{s}=\boldsymbol{t}^{\prime}(s) \boldsymbol{\nu}_{\boldsymbol{t}(s)}$, is a solution in $(0, \bar{T}) \times[0,1)^{d}$ in the periodic sense.

Proof. Let us assume that $s \in C^{1}(0, \bar{T})$ with $s^{\prime}>0$. For every $\bar{\varphi} \in C_{c}^{\infty}\left((0, \bar{T}) ; C^{\infty}\left(\mathbb{T}^{d}\right)\right)$, we define $\varphi_{t}(x):=\bar{\varphi}_{s(t)}(x)$. Therefore, we can write

$$
\begin{aligned}
& \int_{0}^{T} \int_{[0,1)^{d}} \partial_{t} \varphi_{t} d \mu_{t}(x) d t+\frac{1}{2} \int_{0}^{T} \int_{G\left([0,1)^{d}\right)} \bar{\nabla} \varphi_{t}(x, y) d \boldsymbol{\nu}_{t}(x, y) d t \\
& =\int_{0}^{T} \int_{[0,1)^{d}} \partial_{s} \bar{\varphi}_{\boldsymbol{s}(t)}(x) d \mu_{t}(x) \boldsymbol{s}^{\prime}(t) d t+\frac{1}{2} \int_{0}^{T} \int_{G\left([0,1)^{d}\right)} \frac{\bar{\nabla} \bar{\varphi}_{\boldsymbol{s}(t)}(x, y)}{\boldsymbol{s}^{\prime}(t)} d \boldsymbol{\nu}_{t}(x, y) \boldsymbol{s}^{\prime}(t) d t \\
& =\int_{0}^{T} \int_{[0,1)^{d}} \partial_{s} \bar{\varphi}_{s}(x) d \mu_{\boldsymbol{t}(s)}(x) d s+\frac{1}{2} \int_{0}^{\bar{T}} \int_{G\left([0,1)^{d}\right)} \bar{\nabla} \bar{\varphi}_{s}(x, y) \boldsymbol{t}^{\prime}(s) d \boldsymbol{\nu}_{\boldsymbol{t}(s)}(x, y) d s
\end{aligned}
$$

And thus, the pair $\left(\mu_{t}, \boldsymbol{\nu}_{t}\right)_{t \in[0, T]}$ is a solution if and only if $\left(\bar{\mu}_{s}, \overline{\boldsymbol{\nu}}_{s}\right)_{s \in[0, \bar{T}]}$ is also a solution.

\section{The NON-LOCAL TRANSPORT Distance ON $\mathcal{P}\left(\mathbb{T}^{d}\right)$}

In this section we define the periodic non-local Wasserstein metric in the sense of Benamou-Brenier formula and show that in the set where it is finite, it defines a metric that induces a topology stronger than the narrow one (see Definition 2.1).

Definition 5.1. For $\mu_{0}, \mu_{1} \in \mathcal{P}\left(\mathbb{T}^{d}\right)$ we define the function

$$
\mathcal{W}\left(\mu_{0}, \mu_{1}\right)^{2}:=\inf \left\{\int_{0}^{1} \mathcal{A}\left(\mu_{t}, \boldsymbol{\nu}_{t}\right) d t \mid\left(\mu_{t}, \boldsymbol{\nu}_{t}\right)_{t \in[0,1]} \in \mathcal{C} \mathcal{E}_{1}\left(\mu_{0}, \mu_{1}\right)\right\} .
$$

We will show later that this infimum is actually a minimum.

Lemma 5.2. For any $T>0$ we have

$$
\mathcal{W}\left(\mu_{0}, \mu_{1}\right)^{2}=\inf \left\{T \int_{0}^{T} \mathcal{A}\left(\mu_{t}, \boldsymbol{\nu}_{t}\right) d t \mid\left(\mu_{t}, \boldsymbol{\nu}_{t}\right)_{t \in[0,1]} \in \mathcal{C} \mathcal{E}_{T}\left(\mu_{0}, \mu_{1}\right)\right\} .
$$

Proof. Let $s:[0, T] \rightarrow[0,1]$ defined by $s(t)=T^{-1} t$. For any $\left(\mu_{t}, \boldsymbol{\nu}_{t}\right)_{t \in[0,1]} \in \mathcal{C} \mathcal{E}_{1}\left(\mu_{0}, \mu_{1}\right)$ we have, by Lemma 4.4. that $(\bar{\mu}, \overline{\boldsymbol{\nu}}) \in \mathcal{C} \mathcal{E}_{T}\left(\mu_{0}, \mu_{1}\right)$ where $\bar{\mu}_{t}:=\mu_{\boldsymbol{s}(t)}$ and $\overline{\boldsymbol{\nu}}_{t}:=T^{-1} \boldsymbol{\nu}_{\boldsymbol{s}(t)}$. Now, since $\mathcal{A}$ is 2 -homogeneous in the variable $\boldsymbol{\nu}$, we have

$$
\int_{0}^{T} \mathcal{A}\left(\bar{\mu}_{t}, \overline{\boldsymbol{\nu}}_{t}\right) d t=\frac{1}{T^{2}} \int_{0}^{T} \mathcal{A}\left(\mu_{\boldsymbol{s}(t)}, \boldsymbol{\nu}_{\boldsymbol{s}(t)}\right) d t=\frac{1}{T} \int_{0}^{1} \mathcal{A}\left(\mu_{t}, \boldsymbol{\nu}_{t}\right) d t .
$$


Using the inverse $\boldsymbol{s}^{-1}$ we can also see that $\mathcal{C} \mathcal{E}_{T}\left(\mu_{0}, \mu_{1}\right)$ is mapped onto $\mathcal{C} \mathcal{E}_{1}\left(\mu_{0}, \mu_{1}\right)$ with the same relation as above. This concludes the proof.

Lemma 5.3. For any $T>0$ and $\mu_{0}, \mu_{1} \in \mathcal{P}\left(\mathbb{T}^{d}\right)$, we have

$$
\mathcal{W}\left(\mu_{0}, \mu_{1}\right)=\inf \left\{\int_{0}^{T} \sqrt{\mathcal{A}\left(\mu_{t}, \nu_{t}\right)} d t \mid\left(\mu_{t}, \nu_{t}\right)_{t \in[0, T]} \in \mathcal{C} \mathcal{E}_{T}\left(\mu_{0}, \mu_{1}\right)\right\} .
$$

Proof. Let us denote by $\widehat{\mathcal{W}}\left(\mu_{0}, \mu_{1}\right)$ the right-hand side of $(\underline{5.2})$. For every $\left(\mu_{t}, \boldsymbol{\nu}_{t}\right)_{t \in[0,1]} \in \mathcal{C} \mathcal{E}_{1}\left(\mu_{0}, \mu_{1}\right)$ we have by Cauchy-Schwarz inequality that

$$
\int_{0}^{T} \sqrt{\mathcal{A}\left(\mu_{t}, \nu_{t}\right)} d t \leqslant \sqrt{T}\left(\int_{0}^{T} \mathcal{A}\left(\mu_{t}, \nu_{t}\right) d t\right)^{\frac{1}{2}}
$$

and thus, by Lemma 5.2, $\widehat{\mathcal{W}}\left(\mu_{0}, \mu_{1}\right) \leqslant \mathcal{W}\left(\mu_{0}, \mu_{1}\right)$. For the opposite inequality we argue as in [14]. Let us suppose that $\mathcal{W}\left(\mu_{0}, \mu_{1}\right)<\infty$ and let $\left(\mu_{t}, \boldsymbol{\nu}_{t}\right)_{t \in[0,1]} \in \mathcal{C} \mathcal{E}_{1}\left(\mu_{0}, \mu_{1}\right)$ be such that

$$
\int_{0}^{T} \mathcal{A}\left(\mu_{t}, \nu_{t}\right) d t<\infty
$$

Then, for every $\varepsilon>0$ we define the function $\boldsymbol{s}_{\varepsilon}$ on $(0, T)$ by

$$
\boldsymbol{s}_{\varepsilon}(t):=\int_{0}^{t} \sqrt{\varepsilon+\mathcal{A}\left(\mu_{r}, \boldsymbol{\nu}_{r}\right)} d r .
$$

We have that $\boldsymbol{s}_{\varepsilon}$ is strictly increasing and its derivative exists a.e. with $\boldsymbol{s}_{\varepsilon}^{\prime} \geqslant \sqrt{\varepsilon}$. So its inverse $\boldsymbol{t}_{\varepsilon}:=\boldsymbol{s}_{\varepsilon}^{-1}$ is well defined and satisfies the hypothesis from Lemma 4.4. Therefore, we know that $\left(\widehat{\mu}_{s}, \widehat{\boldsymbol{\nu}}_{s}\right)_{s \in\left[0, \boldsymbol{s}_{\varepsilon}(T)\right]} \in$ $\mathcal{C E}_{\boldsymbol{s}_{\varepsilon}(T)}\left(\mu_{0}, \mu_{1}\right)$ where $\widehat{\mu}_{s}:=\mu_{\boldsymbol{t}_{\varepsilon}(s)}$ and $\widehat{\boldsymbol{\nu}}_{s}:=\boldsymbol{t}_{\varepsilon}^{\prime}(s) \boldsymbol{\nu}_{\boldsymbol{t}_{\varepsilon}(s)}$. Using Lemma 5.2, we have

$$
\begin{aligned}
\mathcal{W}\left(\mu_{0}, \mu_{1}\right)^{2} & \leqslant \boldsymbol{s}_{\varepsilon}(T) \int_{0}^{\boldsymbol{s}_{\varepsilon}(T)} \mathcal{A}\left(\widehat{\mu}_{s}, \widehat{\boldsymbol{\nu}}_{s}\right) d s \\
& =\boldsymbol{s}_{\varepsilon}(T) \int_{0}^{\boldsymbol{s}_{\varepsilon}(T)} \boldsymbol{t}_{\varepsilon}^{\prime}(s)^{2} \mathcal{A}\left(\mu_{\boldsymbol{t}_{\varepsilon}(s)}, \boldsymbol{\nu}_{\boldsymbol{t}_{\varepsilon}(s)}\right) d s \\
& =\boldsymbol{s}_{\varepsilon}(T) \int_{0}^{T}\left(\boldsymbol{t}_{\varepsilon}^{\prime} \circ \boldsymbol{s}_{\varepsilon}(t)\right)^{2} \mathcal{A}\left(\mu_{t}, \boldsymbol{\nu}_{t}\right) \boldsymbol{s}_{\varepsilon}^{\prime}(t) d t \\
& =\boldsymbol{s}_{\varepsilon}(T) \int_{0}^{T} \frac{\mathcal{A}\left(\mu_{t}, \boldsymbol{\nu}_{t}\right)}{\varepsilon+\mathcal{A}\left(\mu_{t}, \boldsymbol{\nu}_{t}\right)} \sqrt{\varepsilon+\mathcal{A}\left(\mu_{t}, \boldsymbol{\nu}_{t}\right)} d t \\
& \leqslant \boldsymbol{s}_{\varepsilon}(T)^{2}
\end{aligned}
$$

This holds for all $\varepsilon>0$ and therefore

$$
\mathcal{W}\left(\mu_{0}, \mu_{1}\right)^{2} \leqslant \lim _{\varepsilon \rightarrow 0^{+}} \boldsymbol{s}_{\varepsilon}(T)^{2}=\left(\int_{0}^{T} \mathcal{A}\left(\mu_{t}, \nu_{t}\right) d t\right)^{2}
$$

Since we have this inequality for all pairs $\left(\mu_{t}, \nu_{t}\right)_{t \in[0, T]}$ satisfying (5.3), we can take the infimum over $\mathcal{C} \mathcal{E}_{T}\left(\mu_{0}, \mu_{1}\right)$ and obtain $\mathcal{W}\left(\mu_{0}, \mu_{1}\right) \leqslant \widehat{\mathcal{W}}\left(\mu_{0}, \mu_{1}\right)$.

Proposition 5.4. Given $\mu_{*} \in \mathcal{P}\left(\mathbb{T}^{d}\right)$, the function $\mathcal{W}$ is a metric in $\mathcal{P}_{\mu_{*}}:=\left\{\mu \in \mathcal{P}\left(\mathbb{T}^{d}\right) \mid \mathcal{W}\left(\mu_{*}, \mu\right)<\infty\right\}$ 
Proof. Symmetry: Let $\mu_{0}, \mu_{1} \in \mathcal{P}_{\mu_{*}}$ and $\left(\mu_{t}, \boldsymbol{\nu}_{t}\right)_{t \in[0,1]} \in \mathcal{C} \mathcal{E}_{1}\left(\mu_{0}, \mu_{1}\right)$ and consider the pair $\left(\widetilde{\mu}_{t}, \widetilde{\boldsymbol{\nu}}_{t}\right):=$ $\left(\mu_{1-t},-\boldsymbol{\nu}_{1-t}\right)$. It is straightforward to check that $\left(\widetilde{\mu}_{t}, \widetilde{\boldsymbol{\nu}}_{t}\right) \in \mathcal{C} \mathcal{E}_{1}\left(\mu_{1}, \mu_{0}\right)$ (see Lemma 4.4). Since

$$
\int_{0}^{1} \mathcal{A}\left(\widetilde{\mu}_{t}, \widetilde{\nu}_{t}\right) d t=\int_{0}^{1} \mathcal{A}\left(\mu_{1-t},-\nu_{1-t}\right) d t=\int_{0}^{1} \mathcal{A}\left(\mu_{t}, \nu_{t}\right) d t
$$

we have that

$$
\mathcal{W}\left(\mu_{1}, \mu_{0}\right) \leqslant \mathcal{W}\left(\mu_{0}, \mu_{1}\right)
$$

Analogously, it follows the opposite inequality.

Triangular Inequality: Assume that $\mu_{2} \in \mathcal{P}_{\mu_{*}} . \quad$ Choose $\left(\bar{\mu}_{t}, \overline{\boldsymbol{\nu}}_{t}\right) \in \mathcal{C} \mathcal{E}_{1}\left(\mu_{0}, \mu_{1}\right)$ and $\left(\overline{\bar{\mu}}_{t}, \overline{\overline{\boldsymbol{\nu}}}_{t}\right) \in$ $\mathcal{C E}_{1}\left(\mu_{1}, \mu_{2}\right)$. Define the pair

$$
\left(\widetilde{\mu}_{t}, \widetilde{\boldsymbol{\nu}}_{t}\right)=\left\{\begin{array}{lll}
\left(\bar{\mu}_{2 t}, \overline{\boldsymbol{\nu}}_{2 t}\right) & \text { if } & t \in[0,1 / 2) \\
\left(\overline{\bar{\mu}}_{2 t-1}, \overline{\bar{\nu}}_{2 t-1}\right) & \text { if } & t \in[1 / 2,1]
\end{array} .\right.
$$

Let us to check that $\left(\widetilde{\mu}_{t}, \widetilde{\boldsymbol{\nu}}_{t}\right)$ satisfies the non-local continuity equation in the periodic distributional sense according to Definition 4.1(iii). Let $\varphi \in C_{c}^{\infty}\left((0,1) ; C^{\infty}\left(\mathbb{T}^{d}\right)\right)$, then

$$
\begin{aligned}
& \int_{0}^{1} \int_{[0,1)^{d}} \partial_{t} \varphi_{t} d \widetilde{\mu}_{t}(x) d t+\frac{1}{2} \int_{0}^{1} \int_{[0,1)^{d}} \bar{\nabla} \varphi_{t} d \widetilde{\boldsymbol{\nu}}_{t}(x, y) d t \\
& =\frac{1}{2} \int_{0}^{1} \int_{[0,1)^{d}} \partial_{t} \varphi_{t / 2} d \bar{\mu}_{t}(x) d t+\frac{1}{4} \int_{0}^{1} \int_{[0,1)^{d}} \bar{\nabla} \varphi_{t / 2} d \overline{\boldsymbol{\nu}}_{t}(x, y) d t \\
& +\frac{1}{2} \int_{0}^{1} \int_{[0,1)^{d}} \partial_{t} \varphi_{(t+1) / 2} d \overline{\bar{\mu}}_{t}(x) d t+\frac{1}{4} \int_{0}^{1} \int_{[0,1)^{d}} \bar{\nabla} \varphi_{(t+1) / 2} d \overline{\bar{\nu}}_{t}(x, y) d t \\
& =\frac{1}{2}\left(\int_{[0,1)^{d}} \partial_{t} \varphi_{1 / 2} d \bar{\mu}_{1}-\int_{[0,1)^{d}} \partial_{t} \varphi_{1 / 2} d \overline{\bar{\mu}}_{0}\right)=0,
\end{aligned}
$$

because $\bar{\mu}_{1}=\overline{\bar{\mu}}_{0}=\mu_{1}$. Thus, $\left(\mu_{t}, \boldsymbol{\nu}_{t}\right) \in \mathcal{C} \mathcal{E}_{1}\left(\mu_{0}, \mu_{2}\right)$ and

$$
\mathcal{W}\left(\mu_{0}, \mu_{2}\right) \leqslant \int_{0}^{1} \mathcal{A}\left(\bar{\mu}_{t}, \bar{\nu}_{t}\right) d t+\int_{0}^{1} \mathcal{A}\left(\overline{\bar{\mu}}_{t}, \overline{\bar{\nu}}_{t}\right) d t
$$

Taking the infimum, it follows the triangular inequality.

Now, we assume that $\mathcal{W}\left(\mu_{0}, \mu_{1}\right)=0$. By definition, we can get a sequence $\left(\mu_{t}^{k}, \boldsymbol{\nu}_{t}^{k}\right)_{k \in \mathbb{N}} \in \mathcal{C} \mathcal{E}_{1}\left(\mu_{0}, \mu_{1}\right)$ such that

$$
\lim _{k \rightarrow 0} \int_{0}^{1} \mathcal{A}\left(\mu_{t}^{k}, \nu_{t}^{k}\right) d t=0 .
$$

Now, for any $\varphi \in C\left(\mathbb{T}^{d}\right)$ Lipschitz, we have from Remark 4.3 and from Lemma 3.5 that the following estimate holds for all $k \in \mathbb{N}$ :

$$
\begin{aligned}
\left|\int \varphi d \mu_{1}-\int \varphi d \mu_{0}\right| & \leqslant \frac{1}{2} \int_{0}^{1} \int_{G\left([0,1)^{d}\right)}|\bar{\nabla} \varphi| d\left|\boldsymbol{\nu}_{t}^{k}\right| d t \\
& \leqslant \frac{[\varphi]_{\text {Lip }}}{2} \int_{0}^{1} \int_{G\left([0,1)^{d}\right)} d_{\mathbb{T}^{d}}(x, y) d\left|\boldsymbol{\nu}_{t}^{k}\right| d t \\
& \leqslant C \frac{[\varphi]_{\text {Lip }}}{2} \int_{0}^{1} \sqrt{\mathcal{A}\left(\mu_{t}^{k}, \nu_{t}^{k}\right)} d t \\
& \leqslant C \frac{[\varphi]_{\text {Lip }}}{2}\left(\int_{0}^{1} \mathcal{A}\left(\mu_{t}^{k}, \nu_{t}^{k}\right) d t\right)^{1 / 2}
\end{aligned}
$$

where $[\varphi]_{\text {Lip }}$ is the Lipschitz constant of $\varphi$. Therefore, taking the limit as $k \rightarrow \infty$, we obtain

$$
\int \varphi d \widetilde{\mu}_{0}=\int \varphi d \widetilde{\mu}_{1}
$$


for all Lipschitz $\varphi \in C\left(\mathbb{T}^{d}\right)$. By an approximation argument, we conclude that $\mu_{0}=\mu_{1}$.

For the next result, let us recall the following fact about the Kantorovich-Rubinstein metric (see 22. Theorem 1.14]):

Lemma 5.5. Let $(X, d)$ be a Polish metric space. The Kantorovich-Rubinstein distance on $\mathcal{P}(X)$ is defined by

$$
W_{d}\left(\mu_{0}, \mu_{1}\right):=\inf _{\pi \in \Gamma\left(\mu_{0}, \mu_{1}\right)} \int_{X \times X} d(x, y) d \pi(x, y)
$$

where $\Gamma\left(\mu_{0}, \mu_{1}\right)$ is the set of probability measures $\pi$ on $X \times X$ with marginals $\mu_{0}$ and $\mu_{1}$. Then we have:

i) (Kantorovich-Rubinstein Theorem) Let $\operatorname{Lip}_{1}(X):=\left\{\varphi \in C(X) \mid[\varphi]_{\text {Lip }}<1\right\}$ where

$$
[\varphi]_{\operatorname{Lip}}:=\sup _{x \neq y} \frac{|\varphi(x)-\varphi(y)|}{d(x, y)}
$$

Then

$$
W_{d}\left(\mu_{0}, \mu_{1}\right)=\sup _{\varphi \in \operatorname{Lip}_{1}(x)}\left|\int_{X} \varphi d \mu_{0}-\int_{X} \varphi d \mu_{1}\right|
$$

ii) $W_{d}$ is lower semicontinuous w.r.t. weak (narrow) convergence in $\mathcal{P}(X)$.

Proposition 5.6 (Compactness of solutions). Let $\left\{\left(\mu_{t}^{n}, \boldsymbol{\nu}_{t}^{n}\right)_{t \in[0, T]}\right\}_{n \in \mathbb{N}}$ be a sequence in $\mathcal{C E}_{T}$ such that

$$
S:=\sup _{n \in \mathbb{N}} \int_{0}^{T} \mathcal{A}\left(\mu_{t}^{n}, \boldsymbol{\nu}_{t}^{n}\right) d t<\infty .
$$

Then there exists a subsequence $\left\{\left(\mu_{t}^{n_{k}}, \boldsymbol{\nu}_{t}^{n_{k}}\right)_{t \in[0, T]}\right\}_{k \in \mathbb{N}}$ and a pair $\left(\mu_{t}, \boldsymbol{\nu}_{t}\right)_{t \in[0, T]} \in \mathcal{C} \mathcal{E}_{T}$ such that

$$
\begin{aligned}
& \mu_{t}^{n_{k}} \rightarrow \mu_{t} \text { weakly in } \mathcal{P}\left(\mathbb{T}^{d}\right) \text { for all } t \in[0, T] \\
& \boldsymbol{\nu}^{n_{k}} \stackrel{\boldsymbol{\nu}}{\boldsymbol{\nu}} \text { weakly-* in } \mathcal{M}_{\text {loc }}\left(G\left(\mathbb{T}^{d}\right) \times[0, T]\right)
\end{aligned}
$$

Furthermore, for any convergent sequence of couples $\left\{\left(\mu_{t}^{n}, \boldsymbol{\nu}_{t}^{n}\right)_{t \in[0, T]}\right\}_{n \in \mathbb{N}}$ in the above sense, we have the following lower semicontinuity formula

$$
\int_{0}^{T} \mathcal{A}\left(\mu_{t}, \boldsymbol{\nu}_{t}\right) d t \leqslant \liminf _{n \rightarrow \infty} \int_{0}^{T} \mathcal{A}\left(\mu_{t}^{n}, \boldsymbol{\nu}_{t}^{n}\right) d t
$$

Proof. Let us first define for each $n \in \mathbb{N}$ the measures $d \boldsymbol{\nu}_{n}(x, y, t):=d \boldsymbol{\nu}^{n}(x, y) d t$. So, for every compact subset $K \subset G\left(\mathbb{T}^{d}\right)$ and every Borelian $B \subset[0, T]$, we can use Lemma 3.5 to obtain a constant $C(K)$ depending on $K$ and write

$$
\begin{aligned}
\sup _{n \in \mathbb{N}}\left|\boldsymbol{\nu}_{n}\right|(K \times B) & =\sup _{n \in \mathbb{N}} \int_{B}\left|\boldsymbol{\nu}_{t}^{n}\right|(K) d t \leqslant C(K) \sup _{n \in \mathbb{N}} \int_{B} \sqrt{\mathcal{A}\left(\mu_{t}^{n}, \boldsymbol{\nu}_{t}^{n}\right)} d t \\
& \leqslant C(K)|B|^{1 / 2} \sup _{n \in \mathbb{N}}\left(\int_{0}^{T} \mathcal{A}\left(\mu_{t}^{n}, \boldsymbol{\nu}_{t}^{n}\right) d t\right)^{1 / 2} \\
& \leqslant C(K)|B|^{1 / 2} S^{1 / 2} .
\end{aligned}
$$

Therefore, the measures $\boldsymbol{\nu}_{n}$ have total variation uniformly bounded on each compact subset of $G\left(\mathbb{T}^{d}\right) \times[0, T]$, which assures that we can extract a subsequence $\boldsymbol{\nu}_{n_{k}}$, such that $\boldsymbol{\nu}_{n_{k}} \stackrel{*}{\rightarrow} \boldsymbol{\nu}$ for some $\boldsymbol{\nu} \in \mathcal{M}_{\mathrm{loc}}\left(G\left(\mathbb{T}^{d}\right) \times[0, T]\right)$ as $k \rightarrow \infty$. Furthermore, the estimate (5.5) leads to

$$
\boldsymbol{\nu}(K \times B) \leqslant \int_{B} m_{K}(t) d t, \quad \text { for } m_{K}(t):=\sup _{n \in \mathbb{N}}\left|\boldsymbol{\nu}_{t}^{n}\right|(K) \in L^{1}(0, T),
$$


so, by the Disintegration Theorem (see [3. Theorem 2.28]), there exists a Borel family $\left(\boldsymbol{\nu}_{t}\right)_{t \in[0, T]} \subseteq$ $\mathcal{M}_{\text {loc }}\left(G\left(\mathbb{T}^{d}\right)\right)$ such that $\boldsymbol{\nu}$ can be disintegrated w.r.t. the Lebesgue measure on $[0, T]$ as $d \boldsymbol{\nu}(x, y, t)=$ $d \boldsymbol{\nu}_{t}(x, y) d t$.

Let us show now that the subsequence $\left\{\mu_{t}^{n_{k}}\right\}_{n}$ also admits subsequence which is weakly convergent for all $t \in[0, T]$. For any $k \in \mathbb{N}, 0 \leqslant s \leqslant t \leqslant T$ and $\varphi \in C\left(\mathbb{T}^{d}\right)$ Lipschitz, we can use the same argument as in the proof of Proposition 5.4 to obtain

$$
\begin{aligned}
\left|\int \varphi d \mu_{t}^{n_{k}}-\int \varphi d \mu_{s}^{n_{k}}\right| & \leqslant C \frac{[\varphi]_{\text {Lip }}}{2} \int_{s}^{t} \sqrt{\mathcal{A}\left(\mu_{t}^{n_{k}}, \boldsymbol{\nu}_{t}^{n_{k}}\right)} d t \\
& \leqslant C \frac{[\varphi]_{\text {Lip }}}{2}\left(\int_{0}^{1} \mathcal{A}\left(\mu_{t}^{n_{k}}, \boldsymbol{\nu}_{t}^{n_{k}}\right) d t\right)^{1 / 2} \sqrt{t-s} \\
& \leqslant C \frac{[\varphi]_{\text {Lip }}}{2} S^{1 / 2} \sqrt{t-s}
\end{aligned}
$$

Therefore, by the Kantorovich-Rubinstein Theorem (see Lemma 5.5 (i)), we have that

$$
\limsup _{k \rightarrow \infty} W_{d}\left(\mu_{s}^{n_{k}}, \mu_{t}^{n_{k}}\right) \leqslant C S^{1 / 2}|t-s|^{1 / 2}
$$

Now, by the compactness of $\mathcal{P}\left(\mathbb{T}^{d}\right)$ w.r.t. the weak convergence (Remark 2.2) and the item ii) from Lemma 5.5. we have that the weak topology on $\mathcal{P}\left(\mathbb{T}^{d}\right)$ is compatible with $W_{d}$. This fact together with (5.6) allow us to apply [4. Proposition 3.3.1] to the sequence $\left\{\mu_{t}^{n_{k}}\right\}_{k}$ and obtain that there exists a subsequence, still denoted by $\left\{\mu_{t}^{n_{k}}\right\}_{k}$, and a $W_{d}$-continuous curve $\mu:[0, T] \rightarrow \mathcal{P}\left(\mathbb{T}^{d}\right)$ such that $\mu_{t}^{n_{k}} \rightarrow \mu_{t}$ weakly for all $t \in[0, T]$ as $k \rightarrow \infty$.

In order to see that the pair $\left(\mu_{t}, \boldsymbol{\nu}_{t}\right)_{t \in[0, T]}$ belongs to $\mathcal{C E}_{T}$, we first note that by Lemma 3.5 and the hypothesis above, we have the following estimate

$$
\begin{aligned}
\int_{0}^{t} \int_{G\left([0,1)^{d}\right)} d_{\mathbb{T}^{d}}(x, y) d\left|\boldsymbol{\nu}_{t}\right| d t & \leqslant \sup _{n \in \mathbb{N}} \int_{0}^{t} \int_{G\left([0,1)^{d}\right)} d_{\mathbb{T}^{d}}(x, y) d\left|\boldsymbol{\nu}_{t}^{n}\right| d t \\
& \leqslant C \sup _{n \in \mathbb{N}} \int_{0}^{T} \sqrt{\mathcal{A}\left(\mu_{t}^{n}, \boldsymbol{\nu}_{t}^{n}\right)} d t \\
& \leqslant C T^{1 / 2} \sup _{n \in \mathbb{N}}\left(\int_{0}^{T} \mathcal{A}\left(\mu_{t}^{n}, \boldsymbol{\nu}_{t}^{n}\right) d t\right)^{1 / 2} \\
& \leqslant C T^{1 / 2} S^{1 / 2}<\infty .
\end{aligned}
$$

Also, since $\mu:[0, T] \rightarrow \mathcal{P}\left(\mathbb{T}^{d}\right)$ is $W_{d}$-continuous, it implies that it is weakly continuous as well.

Let us now prove that $\left(\mu_{t}, \boldsymbol{\nu}_{t}\right)_{t \in[0, T]}$ satisfies the continuity equation in the periodic distributional sense. Let $\varphi \in C_{c}^{\infty}\left(0, T ; C^{\infty}\left(\mathbb{T}^{d}\right)\right)$. Then, since $\bar{\nabla} \varphi \notin C_{c}\left(G\left([0,1)^{d}\right) \times[0, T]\right)$, we use an argument by approximation. Let $\eta \in C_{c}^{\infty}(-1,1)$ be such that $0 \leqslant \eta \leqslant 1$ and $\eta(s)=1$ for $|s| \leqslant 1 / 2$ and, for all $\varepsilon>0$, let us define $\eta_{\varepsilon}: G\left([0,1)^{d}\right) \rightarrow[0,1]$ by $\eta_{\varepsilon}(x, y)=\eta\left(d_{\mathbb{T}^{d}}(x, y) / \varepsilon\right)$. Thus, the function $\left(1-\eta_{\varepsilon}\right) \bar{\nabla} \varphi$ does belong to $C_{c}\left(G\left([0,1)^{d}\right) \times[0, T]\right)$ and we have

$$
\lim _{k \rightarrow \infty} \int_{0}^{T} \int_{G\left([0,1)^{d}\right)}\left(1-\eta_{\varepsilon}\right) \bar{\nabla} \varphi d \boldsymbol{\nu}_{t}^{n_{k}} d t=\int_{0}^{T} \int_{G\left([0,1)^{d}\right)}\left(1-\eta_{\varepsilon}\right) \bar{\nabla} \varphi d \boldsymbol{\nu}_{t} d t, \quad \text { for all } \varepsilon>0 .
$$

Let us define now the set $D_{\varepsilon}:=\left\{(x, y) \in G\left([0,1)^{d}\right) \mid d_{\mathbb{T}^{d}}(x, y) \leqslant \varepsilon\right\}$. Since supp $\eta_{\varepsilon} \subseteq D_{\varepsilon}$, we obtain

$$
\begin{aligned}
\left|\int_{0}^{T} \int_{G\left([0,1)^{d}\right)} \eta_{\varepsilon} \bar{\nabla} \varphi_{t} d \boldsymbol{\nu}_{t}^{n_{k}} d t\right| & \leqslant \int_{0}^{T}\left\|\nabla \varphi_{t}\right\|_{\infty} \int_{D_{\varepsilon}} d_{\mathbb{T}^{d}}(x, y) d\left|\boldsymbol{\nu}_{t}^{n_{k}}\right| d t \\
& \leqslant \sup _{t \in[0, T]}\left\|\nabla \varphi_{t}\right\|_{\infty} \int_{0}^{T} \int_{D_{\varepsilon}} d_{\mathbb{T}^{d}}(x, y) d\left|\boldsymbol{\nu}_{t}^{n_{k}}\right| d t .
\end{aligned}
$$


Arguing as in (3.1) with $D_{\varepsilon}$ in the place of $G\left([0,1)^{d}\right)$, we obtain with the inequality (3.2) that

$$
\begin{aligned}
\int_{0}^{T} \int_{D_{\varepsilon}} d_{\mathbb{T}^{d}}(x, y) d\left|\boldsymbol{\nu}_{t}^{n_{k}}\right| d t & \leqslant \sqrt{2} \int_{0}^{T} \sqrt{\mathcal{A}\left(\mu_{t}^{n_{k}}, \boldsymbol{\nu}_{t}^{n_{k}}\right)}\left(\int_{D_{\varepsilon}} d_{\mathbb{T}^{d}}^{2}(x, y) K^{\sigma}(x-y) d y d \mu_{t}^{n_{k}}(x)\right)^{1 / 2} d t \\
& \leqslant \sqrt{2}\left(\int_{0}^{T} \mathcal{A}\left(\mu_{t}^{n_{k}}, \boldsymbol{\nu}_{t}^{n_{k}}\right) d t\right)^{1 / 2} \\
& \times\left(\int_{0}^{T} \int_{D_{\varepsilon}} d_{\mathbb{T}^{d}}^{2}(x, y) K^{\sigma}(x-y) d y d \mu_{t}^{n_{k}}(x) d t\right)^{1 / 2} \\
& \leqslant \sqrt{2} S^{1 / 2} T^{1 / 2}\left(\sup _{x \in[0,1)^{d}} \int_{\{y \mid d(x, y) \leqslant \varepsilon\}} d_{\mathbb{T}^{d}}^{2}(x, y) K^{\sigma}(x-y) d y\right)^{1 / 2} .
\end{aligned}
$$

Since the right-hand side of (5.9) goes to zero as $\varepsilon \rightarrow 0$, we have that

$$
\lim _{\varepsilon \rightarrow 0} \sup _{k \in \mathbb{N}}\left|\int_{0}^{T} \int_{G\left([0,1)^{d}\right)} \eta_{\varepsilon} \bar{\nabla} \varphi_{t} d \boldsymbol{\nu}_{t}^{n_{k}} d t\right|=0 .
$$

Now, since $\left(1-\eta_{\varepsilon}\right) \bar{\nabla} \varphi_{t}(x, y) \rightarrow \bar{\nabla} \varphi_{t}(x, y)$, for all $(x, y) \in G\left([0,1)^{d}\right)$ and $t \in[0, T]$, and

$$
\left|\left(1-\eta_{\varepsilon}\right) \bar{\nabla} \varphi_{t}(x, y)\right| \leqslant \sup _{t \in[0, T]}\left\|\nabla \varphi_{t}\right\|_{\infty} d_{\mathbb{T}^{d}}(x, y) \in L^{1}\left(d \nu_{t} d t\right) \text {, by (15.7), }
$$

we can use the Dominated Convergence Theorem to obtain

$$
\lim _{\varepsilon \rightarrow 0} \int_{0}^{T} \int_{G\left([0,1)^{d}\right)}\left(1-\eta_{\varepsilon}\right) \bar{\nabla} \varphi_{t}(x, y) d \boldsymbol{\nu}_{t} d t=\int_{0}^{T} \int_{G\left([0,1)^{d}\right)} \bar{\nabla} \varphi_{t}(x, y) d \boldsymbol{\nu}_{t} d t
$$

Therefore, from (5.8), (5.10) and (5.11) we conclude that

$$
\lim _{k \rightarrow \infty} \int_{0}^{T} \int_{G\left([0,1)^{d}\right)} \bar{\nabla} \varphi_{t}(x, y) d \boldsymbol{\nu}_{t}^{n_{k}} d t=\int_{0}^{T} \int_{G\left([0,1)^{d}\right)} \bar{\nabla} \varphi_{t}(x, y) d \boldsymbol{\nu}_{t} d t .
$$

Finally, $\mu_{t}^{n_{k}} \rightarrow \mu_{t}$ weakly for all $t \in[0, T]$ implies that

$$
\lim _{k \rightarrow \infty} \int_{0}^{T} \int_{[0,1)^{d}} \partial_{t} \varphi_{t}(x) d \mu_{t}^{n_{k}}(x) d t=\int_{0}^{T} \int_{[0,1)^{d}} \partial_{t} \varphi_{t}(x) d \mu_{t}(x) d t
$$

and this shows that $\left(\mu_{t}, \boldsymbol{\nu}_{t}\right)_{t \in[0, T]}$ satisfies the continuity equation in the periodic distributional sense.

The lower semicontinuity property follows from Lemma 3.3 by considering $\Omega=G\left(\mathbb{T}^{d}\right) \times[0, T]$, the function $f\left(z, h_{1}, h_{2}, h_{3}\right):=\left|h_{3}\right|^{2} \theta_{m}\left(h_{1}, h_{2}\right)^{-1}$ and functional $F:\left(\mathcal{M}_{\text {loc }}\left(G\left(\mathbb{T}^{d}\right) \times[0, T]\right)\right)^{3} \rightarrow[0, \infty]$ defined by

$$
\left(\mu_{t}, \boldsymbol{\nu}_{t}\right)_{t \in[0, T]} \mapsto F\left(\boldsymbol{\mu}_{t}^{1}, \boldsymbol{\mu}_{t}^{2}, \boldsymbol{\nu}_{t}\right):=\int_{0}^{T} \int_{G\left([0,1)^{d}\right)} f\left(\rho_{t}^{1}, \rho_{t}^{2}, w_{t}\right) d \boldsymbol{\lambda}_{t} d t .
$$

Therefore, we have that $F$ is weakly* lower semicontinuous, which gives (5.4) because $\mu_{t}^{n} \rightarrow \mu_{t}$ implies that $\boldsymbol{\mu}_{t}^{i, n} d t \stackrel{*}{\rightarrow} \boldsymbol{\mu}_{t}^{i} d t$ weakly* in $\mathcal{M}_{\mathrm{loc}}\left(G\left(\mathbb{T}^{d}\right) \times[0, T]\right)$.

Proposition 5.7. Let $\mu_{0}, \mu_{1} \in \mathcal{P}\left(\mathbb{T}^{d}\right)$ be such that $\mathcal{W}\left(\mu_{0}, \mu_{1}\right)<\infty$. Then there exists a pair $\left(\mu_{t}, \boldsymbol{\nu}_{t}\right)_{t \in[0,1]} \subset \mathcal{C} \mathcal{E}_{1}\left(\mu_{0}, \mu_{1}\right)$ which attains the infimum in (5.1). Furthermore, any such curve satisfies

$$
\mathcal{A}\left(\mu_{t}, \boldsymbol{\nu}_{t}\right)=\mathcal{W}\left(\mu_{0}, \mu_{1}\right)^{2}, \quad \text { for almost every } t \in[0,1] .
$$

Also, any curve $\left(\mu_{t}\right)_{t \in[0,1]}$ satisfying (5.12) is a constant speed geodesic for $\mathcal{W}$, i.e.

$$
\mathcal{W}\left(\mu_{s}, \mu_{t}\right)=(t-s) \mathcal{W}\left(\mu_{0}, \mu_{1}\right), \text { for all } 0 \leqslant s \leqslant t \leqslant 1 .
$$


Proof. If $\mu_{0}, \mu_{1} \in \mathcal{P}\left(\mathbb{T}^{d}\right)$ are such that $\mathcal{W}\left(\mu_{0}, \mu_{1}\right)<\infty$, then there exists a sequence $\left\{\left(\mu_{t}^{n}, \nu_{t}^{n}\right)_{t \in[0,1]}\right\}_{n \in \mathbb{N}}$ in $\mathcal{C} \mathcal{E}_{1}\left(\mu_{0}, \mu_{1}\right)$ such that

$$
\mathcal{W}\left(\mu_{0}, \mu_{1}\right)^{2}=\lim _{n \rightarrow \infty} \int_{0}^{1} \mathcal{A}\left(\mu_{t}^{n}, \nu_{t}^{n}\right) d t
$$

Thus, by Proposition 5.6. there exists a pair $\left(\mu_{t}, \boldsymbol{\nu}_{t}\right)_{t \in[0,1]} \in \mathcal{C} \mathcal{E}_{1}\left(\mu_{0}, \mu_{1}\right)$ such that, for some subsequence $\left(n_{k}\right)$, we have $\mu_{t}^{n_{k}} \rightarrow \mu_{t}$ weakly in $\mathcal{P}\left(\mathbb{T}^{d}\right)$ for all $t \in[0, T]$ and $\boldsymbol{\nu}^{n_{k}} \rightarrow \boldsymbol{\nu}$ weakly-* in $\mathcal{M}_{\text {loc }}\left(G\left(\mathbb{T}^{d}\right) \times[0, T]\right)$. Therefore, also by the same proposition, we have

$$
\int_{0}^{1} \mathcal{A}\left(\mu_{t}, \boldsymbol{\nu}_{t}\right) d t \leqslant \liminf _{k \rightarrow \infty} \int_{0}^{1} \mathcal{A}\left(\mu_{t}^{n_{k}}, \boldsymbol{\nu}_{t}^{n_{k}}\right) d t=\mathcal{W}\left(\mu_{0}, \mu_{1}\right)^{2} .
$$

Therefore

$$
\mathcal{W}\left(\mu_{0}, \mu_{1}\right)^{2}=\int_{0}^{1} \mathcal{A}\left(\mu_{t}, \nu_{t}\right) d t .
$$

Using Lemma 5.3 and the Cauchy-Schwarz inequality, we conclude that

$$
\mathcal{W}\left(\mu_{0}, \mu_{1}\right) \leqslant \int_{0}^{1} \sqrt{\mathcal{A}\left(\mu_{t}, \boldsymbol{\nu}_{t}\right)} d t \leqslant\left(\int_{0}^{1} \mathcal{A}\left(\mu_{t}, \boldsymbol{\nu}_{t}\right) d t\right)^{\frac{1}{2}}=\mathcal{W}\left(\mu_{0}, \mu_{1}\right) .
$$

This implies that $\mathcal{A}\left(\mu_{t}, \boldsymbol{\nu}_{t}\right)=\mathcal{W}\left(\mu_{0}, \mu_{1}\right)$ for a.e. $t \in[0,1]$.

Moreover, if a pair $\left(\mu_{t}, \boldsymbol{\nu}_{t}\right)_{t \in[0,1]} \subset \mathcal{C} \mathcal{E}_{1}\left(\mu_{0}, \mu_{1}\right)$ is such that $\mathcal{A}\left(\mu_{t}, \boldsymbol{\nu}_{t}\right)$ is constant almost everywhere, we have that, for any $0 \leqslant s \leqslant r \leqslant 1$, the pair $\left(\mu_{t+s}, \boldsymbol{\nu}_{t+s}\right)_{t \in[0, r-s]}$ belongs to $\mathcal{C} \mathcal{E}_{r-s}\left(\mu_{s}, \mu_{r}\right)$ and, by Lemma 5.3 it satisfies

$$
\mathcal{W}\left(\mu_{s}, \mu_{r}\right) \leqslant \int_{0}^{r-s} \mathcal{A}\left(\mu_{t+s}, \nu_{t+s}\right) d t=(r-s) W\left(\mu_{0}, \mu_{1}\right)
$$

The opposite inequality is always true. In fact, if it is not verified for some $s$ and $r$, then

$$
\begin{aligned}
\mathcal{W}\left(\mu_{0}, \mu_{1}\right) & \leqslant \mathcal{W}\left(\mu_{0}, \mu_{s}\right)+\mathcal{W}\left(\mu_{s}, \mu_{r}\right)+\mathcal{W}\left(\mu_{r}, \mu_{1}\right) \\
& <s \mathcal{W}\left(\mu_{0}, \mu_{1}\right)+(r-s) \mathcal{W}\left(\mu_{0}, \mu_{1}\right)+(1-r) \mathcal{W}\left(\mu_{0}, \mu_{1}\right) \\
& =\mathcal{W}\left(\mu_{0}, \mu_{1}\right),
\end{aligned}
$$

which gives a contradiction. Therefore, one must have $\mathcal{W}\left(\mu_{s}, \mu_{r}\right)=(r-s) \mathcal{W}\left(\mu_{0}, \mu_{1}\right)$.

In next proposition we state the convexity of $\mathcal{W}^{2}$ in $\mathcal{P}\left(\mathbb{T}^{d}\right)$. The proof is similar to that of 15 , Proposition 4.7] and is left to the reader.

Proposition 5.8. The function $\left(\mu_{0}, \mu_{1}\right) \in \mathcal{P}\left(\mathbb{T}^{d}\right) \times \mathcal{P}\left(\mathbb{T}^{d}\right) \mapsto \mathcal{W}\left(\mu_{0}, \mu_{1}\right)^{2}$ is convex.

Definition 5.9. For $\mu_{0}, \mu_{1} \in \mathcal{P}\left(\mathbb{T}^{d}\right)$ such that $\mathcal{W}\left(\mu_{0}, \mu_{1}\right)<\infty$, we call any pair $\left(\mu_{t}, \boldsymbol{\nu}_{t}\right)_{t \in[0,1]}$ given by Proposition 5.7 as geodesic pair or optimal pair for $\mathcal{W}\left(\mu_{0}, \mu_{1}\right)$. And the curves $\left(\mu_{t}\right)_{t \in[0,1]}$ as a geodesic curve between $\mu_{0}$ and $\mu_{1}$.

Proposition 5.10. The map $\left(\mu_{0}, \mu_{1}\right) \mapsto \mathcal{W}\left(\mu_{0}, \mu_{1}\right)$ is lower semicontinuous w.r.t. weak convergence. Moreover, the topology induced by $\mathcal{W}$ is stronger than the weak topology.

Proof. Let $\left\{\mu_{0}^{n}\right\}_{n \in \mathbb{N}},\left\{\mu_{1}^{n}\right\}_{n \in \mathbb{N}} \subseteq \mathcal{P}\left(\mathbb{T}^{d}\right)$ be two sequences such that $\mu_{i}^{n} \rightarrow \mu_{i}$ weakly for $i=0,1$ and $\mu_{0}, \mu_{1} \in \mathcal{P}\left(\mathbb{T}^{d}\right)$. Let us suppose that $\liminf _{n \rightarrow \infty} \mathcal{W}\left(\mu_{0}^{n}, \mu_{1}^{n}\right)<\infty$ and let $\left(n_{k}\right)$ be a subsequence such that $\lim _{k \rightarrow \infty} \mathcal{W}\left(\mu_{0}^{n_{k}}, \mu_{1}^{n_{k}}\right)=\liminf _{n \rightarrow \infty} \mathcal{W}\left(\mu_{0}^{n}, \mu_{1}^{n}\right)$. Proposition 5.7 assures that for every $k$ there exists a pair $\left(\mu_{t}^{n_{k}}, \boldsymbol{\nu}_{t}^{n_{k}}\right)_{t \in[0,1]} \in \mathcal{C} \mathcal{E}_{1}\left(\mu_{0}^{n_{k}}, \mu_{1}^{n_{k}}\right)$ such that

$$
\mathcal{W}\left(\mu_{0}^{n_{k}}, \mu_{1}^{n_{k}}\right)^{2}=\int_{0}^{1} \mathcal{A}\left(\mu_{t}^{n_{k}}, \boldsymbol{\nu}_{t}^{n_{k}}\right) d t .
$$


Thus, the existence of the $\operatorname{limit}_{\lim } \mathcal{W}\left(\mu_{0}^{n_{k}}, \mu_{1}^{n_{k}}\right)$ assures that the hypothesis of Proposition 5.6 is valid and we can extract a new subsequence, still denoted by $\left(\mu_{t}^{n_{k}}, \boldsymbol{\nu}_{t}^{n_{k}}\right)_{t \in[0,1]}$, such that $\mu_{t}^{n_{k}} \rightarrow \mu_{t}$ weakly in $\mathcal{P}\left(\mathbb{T}^{d}\right)$ for all $t \in[0, T]$ and $\boldsymbol{\nu}^{n_{k}} \rightarrow \boldsymbol{\nu}$ weakly-* in $\mathcal{M}_{\text {loc }}\left(G\left([0,1)^{d}\right) \times[0, T]\right)$ for some pair $\left(\mu_{t}, \boldsymbol{\nu}_{t}\right)_{t \in[0,1]} \in$ $\mathcal{C} \mathcal{E}_{1}\left(\mu_{0}, \mu_{1}\right)$. Thus, from the lower semicontinuity result in the same proposition, it follows that

$$
\begin{aligned}
\mathcal{W}\left(\mu_{0}, \mu_{1}\right)^{2} & \leqslant \int_{0}^{1} \mathcal{A}\left(\mu_{t}, \boldsymbol{\nu}_{t}\right) d t \leqslant \liminf _{k \rightarrow \infty} \int_{0}^{1} \mathcal{A}\left(\mu_{t}^{n_{k}}, \boldsymbol{\nu}_{t}^{n_{k}}\right) d t=\lim _{k \rightarrow \infty} \mathcal{W}\left(\mu_{0}^{n_{k}}, \mu_{1}^{n_{k}}\right)^{2} \\
& =\liminf _{n \rightarrow \infty} \mathcal{W}\left(\mu_{0}^{n}, \mu_{1}^{n}\right)^{2} .
\end{aligned}
$$

Now, let $\left\{\mu_{n}\right\}_{n}$ be a sequence in $\mathcal{P}\left(\mathbb{T}^{d}\right)$ such that $\mathcal{W}\left(\mu_{n}, \mu\right) \rightarrow 0$. Then, any convergent subsequence $\left\{\mu_{n_{k}}\right\}$ that converges weakly to a $\mu_{*}$ satisfies, by the lower semicontinuity of $\mathcal{W}$, that $\mathcal{W}\left(\mu, \mu_{*}\right) \leqslant$ $\liminf \mathcal{W}\left(\mu_{n_{k}}, \mu\right)=0$. Since $\mathcal{P}\left(\mathbb{T}^{d}\right)$ is sequentially compact, then we obtain that $\mu_{n}$ converges weakly to $\mu$.

Proposition 5.11. Given $\mu_{*} \in \mathcal{P}\left(\mathbb{T}^{d}\right)$, we have that the pair $\left(\mathcal{P}_{\mu_{*}}, \mathcal{W}\right)$ is a complete metric space, where $\mathcal{P}_{\mu_{*}}:=\left\{\mu \in \mathcal{P}\left(\mathbb{T}^{d}\right) \mid \mathcal{W}\left(\mu_{*}, \mu\right)<\infty\right\}$.

Proof. Let $\left(\mu_{n}\right)_{n \in \mathbb{N}} \subseteq \mathcal{P}_{\mu_{*}}$ be a Cauchy sequence w.r.t. $\mathcal{W}$. Since $\mathcal{P}\left(\mathbb{T}^{d}\right)$ is weakly compact, we can extract a subsequence $\left(\mu_{n_{k}}\right)_{k \in \mathbb{N}}$ such that $\mu_{n_{k}} \rightarrow \mu$ for some $\mu \in \mathcal{P}\left(\mathbb{T}^{d}\right)$. Using the lower semicontinuity of $\mathcal{W}$, we have

$$
\mathcal{W}\left(\mu, \mu_{m}\right) \leqslant \liminf _{k \rightarrow \infty} \mathcal{W}\left(\mu_{n_{k}}, \mu_{m}\right), \quad \forall m \in \mathbb{N} .
$$

Thus, since the right hand side of (5.13) can be made arbitrarily small if $m$ is sufficiently big, we conclude that $\left(\mu_{n}\right)_{n \in \mathbb{N}}$ converges to $\mu$, which therefore must belong to $\mathcal{P}_{\mu_{*}}$.

\section{Subdifferential Calculus}

In this section we construct a subdifferential calculus in the space $\mathcal{P}\left(\mathbb{T}^{d}\right)$ endowed with the non-local metric $\mathcal{W}$. We give the corresponding notion of tangent space, subdifferential of a functional and the characterization of this concept to $\lambda$-convex functionals.

Definition 6.1. For $\mu \in \mathcal{P}\left(\mathbb{T}^{d}\right)$ we define the tangent space of $\mathcal{P}\left(\mathbb{T}^{d}\right)$ at $\mu$ by

$$
\operatorname{Tan}_{\mu} \mathcal{P}\left(\mathbb{T}^{d}\right):=\left\{\begin{array}{l|l}
\boldsymbol{\nu} \in \mathcal{M}_{l o c}\left(G\left(\mathbb{T}^{d}\right)\right) \mid & \mathcal{A}(\mu, \boldsymbol{\nu})<\infty \\
& \cdot \mathcal{A}(\mu, \boldsymbol{\nu}) \leqslant \mathcal{A}(\mu, \boldsymbol{\nu}+\eta), \forall \eta \in \mathcal{M}_{\text {loc }}\left(G\left(\mathbb{T}^{d}\right)\right) \text { with } \bar{\nabla} \cdot \eta=0
\end{array}\right\}
$$

If $d \mu(x)=\rho(x) d x$ then

$$
\operatorname{Tan}_{\mu} \mathcal{P}\left(\mathbb{T}^{d}\right):=\left\{w \widehat{\rho}_{m} d K^{\sigma} \in \mathcal{M}_{\text {loc }}\left(G\left(\mathbb{T}^{d}\right)\right) \mid \exists\left(\phi_{n}\right)_{n \in \mathbb{N}} \subset C_{c}^{\infty}\left(\mathbb{T}^{d}\right), \bar{\nabla} \phi_{n} \rightarrow w \text { in } L^{2}\left(\widehat{\rho}_{m} d K^{\sigma}\right)\right\}
$$

Let $\mu_{0}, \mu_{1} \in \mathcal{P}\left(\mathbb{T}^{d}\right)$ be such that $\mathcal{W}\left(\mu_{0}, \mu_{1}\right)<\infty$, then by Proposition 5.7 there exists a geodesic pair $\left(\mu_{t}, \boldsymbol{\nu}_{t}\right)_{t \in[0,1]} \in \mathcal{C} \mathcal{E}_{1}\left(\mu_{0}, \mu_{1}\right)$ satisfying

$$
\mathcal{W}^{2}\left(\mu_{0}, \mu_{1}\right)=\mathcal{A}\left(\mu_{t}, \nu_{t}\right), \quad \text { for a.e. } t \in[0,1] .
$$

If $K \subseteq G\left(\mathbb{T}^{d}\right)$ is compact, then by Lemma 3.5 there exists a constant $C=C(K)$ such that the total variation of $\boldsymbol{\nu}_{t}$ is uniformly bounded for a.e. $t \in[0,1]$ with

$$
\left|\boldsymbol{\nu}_{t}\right|(K) \leqslant C(K) \sqrt{\mathcal{A}\left(\mu_{t}, \boldsymbol{\nu}_{t}\right)}=C(K) \mathcal{W}\left(\mu_{0}, \mu_{1}\right) .
$$

Therefore, there exists a sequence $t_{n} \rightarrow 0^{+}$and $\boldsymbol{\nu} \in \mathcal{M}_{\mathrm{loc}}\left(G\left([0,1)^{d}\right)\right)$ such that $\boldsymbol{\nu}_{t_{n}} \stackrel{*}{\rightarrow} \boldsymbol{\nu}$. Thus, since the set of weak-* limit measures for $\boldsymbol{\nu}_{t}$ as $t \rightarrow 0$ is non empty, we can have the following definition: 
Definition 6.2. Given $\mu_{0}, \mu_{1} \in \mathcal{P}\left(\mathbb{T}^{d}\right)$ such that $\mathcal{W}\left(\mu_{0}, \mu_{1}\right)<\infty$, let $\Gamma_{0}\left(\mu_{0}, \mu_{1}\right)$ be the set of all weak-* limit measures of $\boldsymbol{\nu}_{t}$ as $t \rightarrow 0^{+}$where $\left(\mu_{t}, \boldsymbol{\nu}_{t}\right)_{t \in[0,1]} \in \mathcal{C} \mathcal{E}_{1}\left(\mu_{0}, \mu_{1}\right)$ is a geodesic pair.

Now we give the notion of subdifferential for functionals defined in $\mathcal{P}\left(\mathbb{T}^{d}\right)$ with respect to the geometric structure given by $\mathcal{W}$.

Definition 6.3. Let $\mu \in \mathcal{P}\left(\mathbb{T}^{d}\right)$ with $d \mu(x)=\rho(x) d x$ and let $\mathcal{E}: \mathcal{P}\left(\mathbb{T}^{d}\right) \rightarrow(-\infty,+\infty]$ be a proper and weak lower semicontinuous functional. We say that $d \xi=\zeta \widehat{\rho}_{m} d K^{\sigma} \in \operatorname{Tan}_{\mu} \mathcal{P}\left(\mathbb{T}^{d}\right)$ belongs to the subdifferential $\partial \mathcal{E}(\mu)$ if

$$
\liminf _{\mu_{1} \rightarrow \mu} \frac{1}{\mathcal{W}\left(\mu, \mu_{1}\right)}\left(\mathcal{E}\left(\mu_{1}\right)-\mathcal{E}(\mu)-\inf _{\boldsymbol{\nu} \in \Gamma_{0}\left(\mu, \mu_{1}\right)} \int_{G\left([0,1)^{d}\right)} \zeta(x, y) d \boldsymbol{\nu}(x, y)\right) \geqslant 0
$$

or, equivalently,

$$
\mathcal{E}\left(\mu_{1}\right)-\mathcal{E}(\mu) \geqslant \inf _{\boldsymbol{\nu} \in \Gamma_{0}\left(\mu, \mu_{1}\right)} \int_{G\left([0,1)^{d}\right)} \zeta(x, y) d \boldsymbol{\nu}(x, y)+o\left(\mathcal{W}\left(\mu, \mu_{1}\right)\right) .
$$

The next lemma deals with geodesics constructed via a glueing type process. See [14 for such a type of construction in another context.

Lemma 6.4. Let $\left(\mu_{t}, \boldsymbol{\nu}_{t}\right)_{t \in[0,1]} \in \mathcal{C} \mathcal{E}_{1}\left(\mu_{0}, \mu_{1}\right)$ be a geodesic pair for $\mathcal{W}\left(\mu_{0}, \mu_{1}\right)$ and let $s \in(0,1)$. If $\left(\mu_{t}^{s}, \boldsymbol{\nu}_{t}^{s}\right)_{t \in[0, s]}$ is another geodesic pair for $\mathcal{W}\left(\mu_{0}, \mu_{s}\right)$, then the composition

$$
\bar{\mu}_{t}:=\left\{\begin{array}{ll}
\mu_{t}^{s}, & 0 \leqslant t \leqslant s \\
\mu_{t}, & s<t \leqslant 1
\end{array} \overline{\boldsymbol{\nu}}_{t}:= \begin{cases}\boldsymbol{\nu}_{t}^{s}, & 0 \leqslant t \leqslant s \\
\boldsymbol{\nu}_{t}, & s<t \leqslant 1\end{cases}\right.
$$

is also a geodesic pair for $\mathcal{W}\left(\mu_{0}, \mu_{1}\right)$.

Let us define what a $\lambda$-geodesically convex functional means in our context. We say that a proper functional $\mathcal{E}: \mathcal{P}\left(\mathbb{T}^{d}\right) \rightarrow(-\infty,+\infty]$ is $\lambda$-convex for some $\lambda \in \mathbb{R}$ if for every $\mu_{0}, \mu_{1} \in \mathcal{P}\left(\mathbb{T}^{d}\right)$ with $\mathcal{W}\left(\mu_{0}, \mu_{1}\right)<\infty$, there exists a geodesic curve $\left(\mu_{t}\right)_{t \in[0,1]}$ such that the following inequality is true

$$
\mathcal{E}\left(\mu_{t}\right) \leqslant(1-t) \mathcal{E}\left(\mu_{0}\right)+t \mathcal{E}\left(\mu_{1}\right)-\frac{\lambda}{2} t(1-t) \mathcal{W}\left(\mu_{0}, \mu_{1}\right)^{2}, \quad \forall t \in[0,1] .
$$

In the case when the functional $\mathcal{E}$ is $\lambda$-geodesically convex, we have the following characterization.

Proposition 6.5. Let $\mathcal{E}: \mathcal{P}\left(\mathbb{T}^{d}\right) \rightarrow(-\infty,+\infty]$ be a lower semicontinuous and $\lambda$-geodesically convex functional. If $d \mu(x)=\rho(x) d x$ then $d \xi=\zeta \widehat{\rho}_{m} d K^{\sigma} \in \partial \mathcal{E}(\mu)$, if and only if, for all $\mu_{1} \in \mathcal{P}\left(\mathbb{T}^{d}\right)$ such that $\mathcal{W}\left(\mu, \mu_{1}\right)<\infty$ there exists $\nu_{0} \in \Gamma_{0}\left(\mu, \mu_{1}\right)$ satisfying

$$
\mathcal{E}\left(\mu_{1}\right)-\mathcal{E}(\mu) \geqslant \int_{G\left([0,1)^{d}\right)} \zeta(x, y) d \nu_{0}(x, y)+\frac{\lambda}{2} \mathcal{W}^{2}\left(\mu, \mu_{1}\right) .
$$

Proof. Let $\mu_{1} \in \mathcal{P}\left(\mathbb{T}^{d}\right)$ be such that $\mathcal{W}\left(\mu, \mu_{1}\right)<\infty$ and let $\left(\mu_{t}, \boldsymbol{\nu}_{t}\right)_{t \in[0,1]} \in \mathcal{C} \mathcal{E}_{1}\left(\mu, \mu_{1}\right)$ be a geodesic pair for $\mathcal{W}\left(\mu, \mu_{1}\right)$. Since $\mathcal{W}\left(\mu, \mu_{t}\right)=t \mathcal{W}\left(\mu, \mu_{1}\right)$ for all $t \in[0,1]$, there exists $\boldsymbol{\nu}_{0}^{t} \in \Gamma_{0}\left(\mu, \mu_{t}\right)$ such that

$$
\mathcal{E}\left(\mu_{t}\right)-\mathcal{E}(\mu) \geqslant \int_{G\left([0,1)^{d}\right)} \zeta(x, y) d \boldsymbol{\nu}_{0}^{t}+o(t) .
$$

Claim: $t^{-1} \boldsymbol{\nu}_{0}^{t} \in \Gamma_{0}\left(\mu, \mu_{1}\right)$. 
Let $\left(\mu_{s}^{t}, \boldsymbol{\nu}_{s}^{t}\right)_{s \in[0,1]} \in \mathcal{C} \mathcal{E}_{1}\left(\mu, \mu_{t}\right)$ be a geodesic pair for $\mathcal{W}\left(\mu, \mu_{t}\right)$ such that $\boldsymbol{\nu}_{0}^{t}$ is a limit point of $\left(\boldsymbol{\nu}_{s}^{t}\right)_{s}$ as $s \rightarrow 0^{+}$. By Lemma 4.4, the pair $\left(\mu_{\frac{s}{t}}^{t}, t^{-1} \boldsymbol{\nu}_{\frac{s}{t}}^{t}\right)_{s \in[0, t]}$ are still geodesic pairs for $\mathcal{W}\left(\mu, \mu_{t}\right)$ for all $t$ and, by Lemma 6.4, the pairs $\left(\bar{\mu}_{s}^{t}, \overline{\boldsymbol{\nu}}_{s}^{t}\right)_{s \in[0,1]}$ defined by

$$
\bar{\mu}_{s}^{t}:=\left\{\begin{array}{cc}
\mu_{\frac{s}{t}}^{t}, & 0 \leqslant s \leqslant t \\
\mu_{s}, & t<s \leqslant 1
\end{array} \overline{\boldsymbol{\nu}}_{s}^{t}:=\left\{\begin{array}{cc}
t^{-1} \boldsymbol{\nu}_{\frac{s}{t}}^{t}, & 0 \leqslant s \leqslant t \\
\boldsymbol{\nu}_{s}, & t<s \leqslant 1
\end{array}\right.\right.
$$

are also geodesic pairs for $\mathcal{W}\left(\mu, \mu_{1}\right)$ and have $t^{-1} \boldsymbol{\nu}_{0}^{t}$ as a limit point for $\overline{\boldsymbol{\nu}}_{s}$ as $s \rightarrow 0^{+}$.

Now, by Proposition [5.7, these geodesic pairs satisfy $W^{2}\left(\mu, \mu_{1}\right)=\mathcal{A}\left(\bar{\mu}_{s}^{t}, \bar{\nu}_{s}^{t}\right)$ for all $t \in[0,1]$ and for a.e. $s \in[0,1]$, and for all $K \subseteq G\left(\mathbb{T}^{d}\right)$ we can use Lemma 3.5 and the weak-weak* lower semicontinuity of $\mathcal{A}$ to estimate

$$
t^{-1}\left|\boldsymbol{\nu}_{0}^{t}\right|(K) \leqslant C(K) \sqrt{\mathcal{A}\left(\mu, t^{-1} \boldsymbol{\nu}_{0}^{t}\right)} \leqslant C(K) \liminf _{s \rightarrow 0} \sqrt{\mathcal{A}\left(\bar{\mu}_{s}^{t}, \overline{\boldsymbol{\nu}}_{s}^{t}\right)}=C(K) \mathcal{W}\left(\mu, \mu_{1}\right) .
$$

Therefore, there exists $\boldsymbol{\nu}_{0}$ weak-* limit point of $t^{-1} \boldsymbol{\nu}_{0}^{t}$ as $t \rightarrow 0^{+}$. Finally, by Proposition 5.6 there exists also a geodesic pair $\left(\overline{\bar{\mu}}_{t}, \overline{\overline{\boldsymbol{\nu}}}_{t}\right)_{t \in[0,1]} \in \mathcal{C} \mathcal{E}_{1}\left(\mu, \mu_{1}\right)$ such that $\overline{\overline{\boldsymbol{\nu}}}_{t} \stackrel{*}{\rightarrow} \boldsymbol{\nu}_{0}$ and therefore, $\boldsymbol{\nu}_{0} \in \Gamma_{0}\left(\mu, \mu_{0}\right)$.

Since $\mathcal{E}$ is $\lambda$-convex, the following inequality holds for all $t \in(0,1]$

$$
\mathcal{E}\left(\mu_{1}\right)-\mathcal{E}(\mu)-\frac{\lambda}{2}(1-t) \mathcal{W}^{2}\left(\mu, \mu_{1}\right) \geqslant \frac{\mathcal{E}\left(\mu_{t}\right)-\mathcal{E}(\mu)}{t}
$$

and, for $d \xi=\zeta \widehat{\rho}_{m} d K^{\sigma} \in \partial \mathcal{E}(\mu)$, we obtain from (6.1) that

$$
\liminf _{t \rightarrow 0} \frac{\mathcal{E}\left(\mu_{t}\right)-\mathcal{E}(\mu)}{t} \geqslant \liminf _{t \rightarrow 0} \int_{G\left([0,1)^{d}\right)} \zeta(x, y) t^{-1} d \boldsymbol{\nu}_{0}^{t}=\int_{G\left([0,1)^{d}\right)} \zeta(x, y) d \boldsymbol{\nu}_{0},
$$

where the equality follows from the following fact: since $d \mu(x)=\rho(x) d x$ and $\mathcal{A}\left(\mu, t^{-1} \boldsymbol{\nu}_{0}^{t}\right), \mathcal{A}\left(\mu, \boldsymbol{\nu}_{0}\right)<\infty$ then there exist $w_{0}^{t}, w_{0} \in L^{2}\left(\widehat{\rho}_{m} d K^{\sigma}\right)$ such that $t^{-1} d \boldsymbol{\nu}_{0}^{t}=w_{0}^{t} \widehat{\rho}_{m} d K^{\sigma}$ and $d \boldsymbol{\nu}_{0}=w_{0} \widehat{\rho}_{m} d K^{\sigma}$. Therefore, $t^{-1} \boldsymbol{\nu}_{0}^{t} \stackrel{*}{\rightarrow} \boldsymbol{\nu}_{0}$ implies that $w_{0}^{t} \rightarrow w_{0}$ in $L^{2}\left(\widehat{\rho}_{m} d K^{\sigma}\right)$. Thus, since $\zeta \in L^{2}\left(\widehat{\rho}_{m} d K^{\sigma}\right)$, we have

$$
\begin{aligned}
\lim _{t \rightarrow 0} \int_{G\left([0,1)^{d}\right)} \zeta(x, y) t^{-1} d \boldsymbol{\nu}_{0}^{t} & =\lim _{t \rightarrow 0} \int_{G\left([0,1)^{d}\right)} \zeta(x, y) w_{0}^{t}(x, y) \widehat{\rho}_{m} d K^{\sigma} \\
& =\int_{G\left([0,1)^{d}\right)} \zeta(x, y) w_{0}(x, y) \widehat{\rho}_{m} d K^{\sigma} \\
& =\int_{G\left([0,1)^{d}\right)} \zeta(x, y) d \boldsymbol{\nu}_{0} .
\end{aligned}
$$

Finally, from (6.2) and (6.3) we obtain the desired expression.

Remark 6.6. If $\Omega \subset \mathbb{R}^{k}$ is open and $\boldsymbol{\nu}_{n} \stackrel{*}{\rightarrow} \boldsymbol{\nu}$ in $\mathcal{M}_{\text {loc }}(\Omega)$ with $d \boldsymbol{\nu}_{n}=w_{n} d \boldsymbol{\pi}$ for some $0 \leqslant \pi \in \mathcal{M}_{\text {loc }}(\Omega)$, then $w_{n} \rightarrow w$ in $L^{2}(d \boldsymbol{\pi})$.

\section{Generalized minimizing Movements}

In this section we consider the Rényi entropy functional which is the internal energy functional associated to the porous medium equation in the classical case. We show that for each $\mu_{0} \in \mathcal{P}\left(\mathbb{T}^{d}\right)$ there exists an absolutely continuous curve obtained as the limit (up to a subsequence) of the discrete variational scheme (1.3). As commented in the introduction, such curve is a generalized minimizing movement for (1.1) starting at $\mu_{0}$. 
Definition 7.1. For $\mu \in \mathcal{P}\left(\mathbb{T}^{d}\right)$ and $m \in(0,2]$ we define the entropy $\mathcal{U}_{m}$ at $\mu$ by

$$
\mathcal{U}_{m}(\mu):= \begin{cases}\int_{[0,1)^{d}} U_{m}(\rho(x)) d x, & \text { if } d \mu=\rho(x) d x \\ +\infty, & \text { else }\end{cases}
$$

where

$$
U_{m}(s):=\left\{\begin{array}{ll}
s \log _{m} & \text { if } m=1 \\
\frac{s^{m}}{m-1} & \text { else }
\end{array} .\right.
$$

Definition 7.2. For $\mu \in \mathcal{P}\left(\mathbb{T}^{d}\right)$ and $m=1$ we define the entropy dissipation (or fisher information associated) $\mathcal{I}_{1}$ at $\mu$ by

$$
\mathcal{I}_{1}(\mu):=\Xi\left(\boldsymbol{\mu}^{1}, \boldsymbol{\mu}^{2}\right):=\frac{1}{2} \int_{G\left([0,1)^{d}\right)} \frac{\left(\rho^{1}-\rho^{2}\right)^{2}}{\theta\left(\rho^{1}, \rho^{2}\right)} d \boldsymbol{\lambda}
$$

where $d \boldsymbol{\mu}^{1}(x, y)=K^{\sigma}(x-y) d y d \mu(x), d \boldsymbol{\mu}^{2}(x, y)=K^{\sigma}(x-y) d x d \mu(y)$ and $\lambda \in \mathcal{M}_{\text {loc }}\left(G\left(\mathbb{T}^{d}\right)\right)$ is such that $\boldsymbol{\mu}^{1}, \boldsymbol{\mu}^{2} \ll \lambda$.

Remark 7.3. Note that when $d \mu(x)=\rho(x) d x$, we have

$$
\mathcal{I}_{1}(\mu)=\frac{1}{2} \int_{G\left([0,1)^{d}\right)} \bar{\nabla} \rho(x, y) \bar{\nabla} \log \rho(x, y) K^{\sigma}(x-y) d x d y .
$$

Since the integrand in the definition of $\Xi$ is a lower semicontinuous function of $\rho^{1}$ and $\rho^{2}$, and also positively 1-homogeneous, we have that $\Xi$ is lower semicontinuous w.r.t. the weak ${ }^{*}$ convergence in $\mathcal{M}_{\text {loc }}\left(G\left(\mathbb{T}^{d}\right)\right)$. Therefore, if $\mu_{n} \rightarrow \mu$ weakly in $\mathcal{P}\left(\mathbb{T}^{d}\right)$, then $\boldsymbol{\mu}_{n}^{i} \stackrel{*}{\rightarrow} \boldsymbol{\mu}^{i}$, for $i=1,2$, and it implies that

$$
\mathcal{I}_{1}(\mu)=\Xi\left(\boldsymbol{\mu}^{1}, \boldsymbol{\mu}^{2}\right) \leqslant \liminf _{n \rightarrow \infty} \Xi\left(\boldsymbol{\mu}_{n}^{1}, \boldsymbol{\mu}_{n}^{2}\right)=\liminf _{n \rightarrow \infty} \mathcal{I}_{1}\left(\mu_{n}\right) .
$$

Analogously, for $m \neq 1$ we can define the fisher information as

$$
\mathcal{I}_{m}(\mu):=\frac{m}{m-1} \int_{G\left([0,1)^{d}\right)} \bar{\nabla}\left(\rho^{m-1}\right) \bar{\nabla}\left(\rho^{m}\right) K^{\sigma}(x-y) d x d y,
$$

for $d \mu=\rho d x$. Note that, in this case the integrand is $(2 m-1)-$ homogeneous and thus it is not possible to extend it for more general measures.

The next theorem shows a coercivity property to the internal energy defined in Definition 7.1 .

Theorem 7.4. For any $\tau>0$ and $\mu_{*} \in \mathcal{P}\left(\mathbb{T}^{d}\right)$, the function

$$
\mu \in \mathcal{P}_{\mu_{*}} \mapsto \Phi\left(\tau, \mu_{*} ; \mu\right):=\frac{1}{2 \tau} \mathcal{W}^{2}\left(\mu_{*}, \mu\right)+\mathcal{U}_{m}(\mu)
$$

is bounded from below in $\mathcal{P}\left(\mathbb{T}^{d}\right)$. Moreover, there exists a unique $\mu^{0} \in \mathcal{P}\left(\mathbb{T}^{d}\right)$ (depending on $\tau$ and $\mu_{*}$ ) such that

$$
\Phi\left(\tau, \mu_{*} ; \mu^{0}\right) \leqslant \Phi\left(\tau, \mu_{*} ; \mu\right), \quad \forall \mu \in \mathcal{P}\left(\mathbb{T}^{d}\right) .
$$

Proof. We first notice that for $m=1$ and any $\mu=\rho(x) d x \in \mathcal{P}_{a c}\left(\mathbb{T}^{d}\right)$, we can rewrite $\mathcal{U}_{1}$ as

$$
\mathcal{U}_{1}(\mu)=\int_{[0,1)^{d}} \rho \log \rho d x=\int_{[0,1)^{d}}(\rho \log \rho-\rho+1) d x
$$

and use the fact that $s \mapsto s \log s-s+1$ is nonnegative in $[0, \infty)$.

If $m \in(1,2]$ then the nonnegativity of $\mathcal{U}_{m}$ is clear. 
Finally, if $m \in(0,1)$, we have that

$$
\mathcal{U}_{m}(\mu)=\int_{[0,1)^{d}}\left(\frac{\rho(x)^{m}}{m-1}+\rho(x)-1\right) d x
$$

and we can use the fact that $s \mapsto \frac{s^{m}}{m-1}+s-1$ is bounded from below.

Therefore, for all $m \in(0,2], \tau>0$ and $\mu_{*} \in \mathcal{P}\left(\mathbb{T}^{d}\right)$, the functional $\Phi$ is bounded from below.

The existence of points of minimum follows from the fact that any minimizing sequence for $\Phi$ is relatively compact w.r.t. the weak convergence. Therefore, by the lower semicontinuity of $\mathcal{W}$ and $\mathcal{U}_{m}$ (the semicontinuity of the functional w.r.t. the weak convergence is a very well known fact), we have that any limit point attains the minimum value for $\Phi$. The uniqueness is a consequence of the fact that the composition $t \in[0,1] \mapsto \mu_{t}:=(1-t) \mu_{0}+t \mu_{1} \mapsto \mathcal{U}_{m}\left(\mu_{t}\right)$ is strictly convex.

Theorem 7.5. For any $\tau>0$ and $\mu_{0} \in \mathcal{P}\left(\mathbb{T}^{d}\right)$ such that $\mathcal{U}_{m}\left(\mu_{0}\right)<\infty$ we can define

$$
\mu_{\tau}^{0}:=\mu_{0} \quad \text { and } \quad \mu_{\tau}^{n}:=\operatorname{argmin}\left\{\Phi\left(\tau, \mu_{0}^{n-1} ; \mu\right) \mid \mu \in \mathcal{P}\left(\mathbb{T}^{d}\right)\right\}, \forall n \in \mathbb{N} .
$$

Now defining the interpolation $\mu_{\tau}:[0, \infty) \rightarrow \mathcal{P}\left(\mathbb{T}^{d}\right)$ by

$$
\mu_{\tau}(t):=\mu_{\tau}^{n} \quad \text { for } \quad t \in[n \tau,(n+1) \tau), \quad \text { and } n \in \mathbb{N} \cup\{0\}
$$

we have that there exists a curve $\mu \in A C_{\mathrm{loc}}\left([0, \infty), \mathcal{P}\left(\mathbb{T}^{d}\right)\right)$ such that (up to a subsequence)

$$
\mu_{\tau}(t) \rightarrow \mu(t), \text { as } \tau \rightarrow 0 \forall t \geqslant 0
$$

Proof. For a fixed $\mu_{*} \in \mathcal{P}\left(\mathbb{T}^{d}\right)$, we have from the previous results that the following ones are true:

1. The weak topology on $\mathcal{P}_{\mu_{*}}$ is compatible with $\mathcal{W}$, in the sense that it is weaker than the one generated by $\mathcal{W}$ and the function $\left(\mu_{0}, \mu_{1}\right) \mapsto \mathcal{W}\left(\mu_{0}, \mu_{1}\right)$ is weak lower semicontinuous;

2. The entropy functional is weak lower semicontinuous on $\mathcal{P}_{\mu_{*}}$;

3. For all $\tau>0$ the functional $\mu \in \mathcal{P}_{\mu_{*}} \mapsto \Phi\left(\tau, \mu_{*} ; \mu\right)$ is bounded from below;

4. The space $\mathcal{P}_{\mu_{*}}$ is weakly sequentially compact.

Therefore, we just need to apply the general results for metric spaces like in 4, Proposition 2.2.3 or Corollary 3.3.4].

Acknowledgement: LCFF was supported by CNPq and FAPESP, Brazil. MCS acknowledges the support from the São Paulo Research Foundation (FAPESP) grant \#2014/23326-1, Brazil. JCV-G acknowledges the support from CNPq, Brazil.

\section{REFERENCES}

[1] S. Abe, S. Thurner. Anomalous diffusion in view of Einstein's 1905 theory of Brownian motion, Physica A: Statistical Mechanics and its Applications, Volume 356, Issues 2-4, 15 October 2005, Pages 403-407, ISSN 0378-4371.

[2] M. Agueh, Existence of solutions to degenerate parabolic equations via the Monge-Kantorovich theory, Adv. Differential Equations 10 (3) (2005), 309-360. 
[3] L. Ambrosio, N. Fusco, D. Pallara. Functions of bounded variation and free discontinuity problems. vol. 254, Clarendon Press Oxford, 2000.

[4] L. Ambrosio, N. Gigli, G. Savaré. Gradient flows in metric spaces and in the space of probability measures. Lectures in Mathematics ETH Zürich. Birkhäuser Verlag, Basel, second edition, 2008.

[5] I. Athanasopoulos, L. A. Caffarelli. Continuity of the temperature in boundary heat control problems, Adv. Math. 224 (2010) 293-315.

[6] J.-D. Benamou, Y. Brenier. A computational fluid mechanics solution to the Monge-Kantorovich mass transfer problem, Numer. Math. 84 (2000), no. 3, 375-393.

[7] A. Blanchet, P. Laurençot, The parabolic-parabolic Keller-Segel system with critical diffusion as a gradient flow in $\mathbb{R}^{d}, d \geqslant 3$. Comm. Partial Differential Equations 38 (2013), no. 4, 658-686.

[8] G. Buttazzo. Semicontinuity, relaxation and integral representation in the calculus of variations, Pitman Research Notes in Mathematics Series, 207. Longman Scientific \& Technical, Harlow, 1989.

[9] J. A. Carrillo, M. Di Francesco, A. Figalli, T. Laurent, D. Slepcev, Global-in-time weak measure solutions and finite-time aggregation for nonlocal interaction equations, Duke Math. J. 156 (2011), no. 2, 229-271.

[10] J. A. Carrillo, D. Slepcev. Example of a displacement convex functional of first order. Calc. Var. Partial Differential Equations 36 (2009), no. 4, 547-564.

[11] A. de Pablo, F. Quiros, A. Rodriguez, J.L. Vazquez. A fractional porous medium equation. Adv. Math. 226 (2011), 1378-1409.

[12] A. de Pablo, F. Quiros, A. Rodriguez, J.L. Vazquez. A general fractional porous medium equation. Comm. Pure Appl. Math. 65 (2012), no. 9, 1242-1284.

[13] M. Di Francesco, S. Fagioli, Measure solutions for non-local interaction PDEs with two species, Nonlinearity 26 (2013), no. 10, 2777-2808.

[14] J. Dolbeault, B. Nazaret, G. Savaré. A new class of transport distances between measures. Calc. Var. Partial Differential Equations 34 (2009), no. 2, 193-231.

[15] M. Erbar, Gradient flows of the entropy for jump processes. Ann. Inst. Henri Poincaré Probab. Stat. 50 (2014), no. 3, 920-945.

[16] L. C. F. Ferreira, J. C. Valencia-Guevara. Periodic solutions for a 1D-model with nonlocal velocity via mass transport, to appear in J. Diff. Eq. (2016).

[17] W. Gangbo, A. Tudorascu. Weak KAM theory on the Wasserstein torus with multidimensional underlying space. Comm. Pure Appl. Math. 67 (2014), no. 3, 408-463.

[18] M. Jara. Hydrodynamic limit of particle systems with long jumps, preprint, http://arxiv.org/abs/0805.1326

[19] R. Jordan, D. Kinderlehrer, F. Otto. The variational formulation of the Fokker-Planck equation. SIAM J. Math. Anal. 29 (1998), no. 1, 1-17.

[20] F. Otto. The geometry of dissipative evolution equations: the porous medium equation. Comm. Partial Differential Equations 26 (2001), no. 1-2, 101-174.

[21] L. Roncal, P. Stinga. Fractional Laplacian on the torus, to appear in Commun. Contemp. Math. (2015).

[22] C. Villani. Topics in optimal transportation. Graduate Studies in Mathematics 58, American Mathematical Society, Providence, RI, 2003.

[23] L. Vlahos, H. Isliker, Y. Kominis, K. Hizonidis. Normal and anomalous Diffusion: A tutorial, in: T. Bountis (Ed.), Order and Chaos, vol. 10, Patras University Press, 2008.

[24] H Weitzner, G.M Zaslavsky, Some applications of fractional equations, Communications in Nonlinear Science and Numerical Simulation, Volume 8, Issues 3-4, September-December 2003, Pages 273-281, ISSN 1007-5704. 